\title{
A CLASS OF SEQUENCES OF FUNCTIONS
}

\author{
BY
}

D. G. BOURGIN

1. Introduction. A recent article [1] treated the characterization of functions in $L_{2}(-\pi, \pi)$ which are odd, periodic of period $2 \pi$, and such that $\{f(n x)\}$ is orthonormal $\left({ }^{1}\right)$. Part of the present paper is devoted to these questions also. However, with the exception of a few results given in sharper form here than in [1] the theorems obtained are new in content. Largely due to the exploitation of different methods, it has been possible to develop new aspects and to make significant advances over previous results. The theory of the main problem is now well integrated, and the general features clearly defined. Our methods extend to cognate problems. We need only cite the results obtained in $\$ 9$ on an important problem.

As partial illustration of the remarks above, we give an abbreviated survey of some of the results obtained. The numbers refer to sections. In (2) we show that, in general, theorems proved heretofore for $\phi(z) \in K^{\prime}$ are valid also for $\phi(z) \in K^{U}$. Moreover, if $\phi(z) \in K^{U}$ the convergence abscissa of the Dirichlet expansion lies to the left of $R(z)=0$. In (3) we establish the basic necessity conditions for $\phi(z) \in K$. In (4), besides other results, we generalize Theorems $7.4,10.3$ and 10.7 of [1]. In (5) the central theorem for $\phi(z) \in K^{\prime}$ is that finiteness of base implies $\phi(z)$ is a quasi-elementary solution. A weak extension to the case of nonfinite bases is included. If $\phi(z) \in K^{\prime}$, then the elements of the base of cardinal number $\alpha$ may be replaced by those of any other base with cardinal $\beta, \beta \leqq \alpha$. In (6) we give a key theorem covering necessary and sufficient conditions for a solution in terms of the associated Dirichlet series. In (7) we establish a close relationship between Watson transforms and solutions of our main problem. For solutions in $K^{\prime}$ or solution with a oneelement base, the correspondence is exact. In (8) we give two types of constructive examples for which $R(z)=0$ is a natural boundary for a solution in $K$. In (9) we turn to a new problem, namely that of the completeness of sequences $\{f(n x)\}$ in $L_{2}(0 ;-\pi, \pi)$ where orthogonality restrictions are waived. Our most interesting results are perhaps Theorems 9.7 and 9.8 , for the situation covered is quite outside the range of the Paley-Wiener ideas. This is supported by an example. In (10) we give a general setting to the questions concerning us in the first 8 sections. A special case is that of $\sum a_{n} a_{n+k}=\delta_{0 k}$. There is an isomorphism with solutions in $K$ for a one-element base. For this special situation stronger statements can be made than in the general case.

Presented to the Society, November 25, 1945, under the titles $A$ class of generating functions and Complete sets of functions; received by the editors Deceniber 4, 1945.

(1) Numbers in brackets refer to the bibliography. 
A number of open problems, connected with completing and extending the ideas of this paper, are mentioned in the remarks.

We mention some of our principal conventions. The terminology is consistent with that of $[1]$. We write $[f(x), g(x)]$ for $\pi^{-1} \int_{-\pi}^{\pi} f(x) g(x) d x$ and the norm, $\|f(x)\|$, is $[f(x), f(x)]^{1 / 2}$. We indicate the Hilbert space of odd functions in $L_{2}(-\pi, \pi)$ by $L_{2}(0 ;-\pi, \pi)$. Unless explicit statement is made to the contrary, functions of $x$ are understood to be periodic of period $2 \pi$ and in $L_{2}(0 ;-\pi, \pi)$. We use O.N. as an abbreviation for orthonormal. We shall deal with functions of the real variable $f(x) \sim \sum a_{n} \sin n x$ with real sequences $\left\{a_{n}\right\}$ and with the Dirichlet expansion $\phi(z) \backsim \sum a_{n} n^{-z}$. We say here that $f(x),\left\{a_{n}\right\}$, and $\phi(z)$ are associated. Compactness of exposition is obtained by using the same class symbol for each type of entity. Thus $K$ is (a) the class of all (odd) ( $\left.{ }^{2}\right)$ functions $f(x)$ which are periodic of period $2 \pi$ and satisfy $I$, (b) the class of all sequences $\left\{a_{n}\right\} \in l_{2}$ satisfying II, (c) the class of all $\phi(z)$ whose coefficients $a_{n}$ are in (b). Besides the sequence spaces $l_{1}$ and $l_{2}$ we use a subset $U$ of $l_{2}$ consisting of sequences $\left\{a_{n}\right\}$ for which $\sum a_{n} n^{i y}$ converges uniformly. We write $K^{\prime}$ for the subclass of $K$ for which $\left\{a_{n}\right\} \in l_{1}$ and $K^{U}$ for the subclass of $K$ for which $\left\{a_{n}\right\} \in U$. Evidently $K^{U} \supset K^{\prime}$. We sometimes refer to the members of these classes as solutions. We abbreviate "almost periodic" in the sense of Bohr to a.p., and "almost everywhere in the (Lebesgue) measure sense," to a.e. We express

$$
L_{T \rightarrow \infty} \frac{1}{2 T} \int_{-T}^{T}|Q(s+i t)|^{2} d t
$$

by $M\{Q(s+i t)\}$. The symbol $(r, s)$ denotes the G.C.D. of the integers $r$ and $s$ and $r \mid s$ indicates that $r$ is a divisor of $s$. We make the convention that $a_{t}=0$ if $t$ is not an integer. Let $S$ be a subset of the positive integers. If every element of $S$ can be expressed in the form

$$
\prod_{j=1}^{N} p_{j}^{l(j)}
$$

where $l(j)$ is a non-negative integer and the $\left\{p_{i}\right\}$ are primes, we say that $S$ has the base $\left\{p_{i}\right\}$. The terms "finite base" and "nonfinite base" are then clear. If $a_{n}$ vanishes except for $n$ in a set with the base $\left\{p_{j}\right\}$, we say $\left\{a_{n}\right\}$ has the base $\left\{p_{j}\right\}$; similarly we refer to $f(x)$ or $\phi(z)$ as having this base.

The main part of the paper may be read without previous knowledge of [1]. There are, however, certain relations which play a central role in both papers. These are repeated below with the same number designation used in [1].

$$
[f(n x), f(m x)]=\delta_{n m}
$$

(2) This is not a necessary restriction, cf. [1, footnote 1$]$. 


$$
\sum_{k=1}^{\infty} a_{k} a_{m k / n}=\delta_{n m}
$$

III

$$
\phi(z) \phi(-z)=1 \text {. }
$$

A quasi elementary solution $\phi(z)$ has the form

$$
M^{-z}\left(1+\sum_{j=1}^{N} h_{j} n_{j}^{2}\right) /\left(1+\sum_{j=1}^{N} h_{j} n_{i}^{-z}\right),
$$

where $n_{j} \mid j=1, \cdots, N$ is an ascending sequence of integers, $M$ is a multiple of the L.C.M. of the $n_{j}$ 's, $\left\{h_{j} \mid j=1, \cdots, N\right\}$ is a real finite sequence and the denominator does not vanish for $R(z) \geqq 0$. This is formally a somewhat less restrictive definition than that given in [1]. If $N=1$ we have an elementary solution.

2. The class $K^{v}$. In our previous work special significance was attached to the case $\left\{a_{n}\right\} \in K^{\prime}$. It turns out that the basic theorems for this class retain their validity when $K^{\prime}$ is replaced by $K^{U}$. The reason for this is that the associated Dirichlet series represents an a.p. function for $R(z)=0$ and the combination properties of almost periodic functions prove sufficient to supplant the more restrictive requirement of absolute convergence.

LEMMA 2.1. If $\sum a_{n} n^{-i t}$ converges uniformly, then $\sum a_{n} n^{-s}$ converges uniformly for $R(z) \geqq 0$.

An application of Abel's theorem along conventional lines to the series $\sum\left(a_{n} n^{-s}\right) n^{-i t}$ leads to a proof.

THEOREM 2.1. The relation $\left\{a_{n}\right\} \in K^{U}$ implies and is implied by $\left\{a_{n}\right\} \in U$ and $\left|\sum a_{n} n^{i t}\right|=1$.

Evidently $\phi(i t)=\sum a_{n} n^{-i t}$ is a.p. It follows that $\phi(i t) \phi(-i t)$ is also a.p. $[2$, p. 6] and has the generalized Fourier series

$$
2^{-1} \sum_{(m, n)=1}\left((m / n)^{i t}+(m / n)^{-i t}\right) \sum_{k=1}^{\infty} a_{k m} a_{k n}=1 .
$$

Hence $\phi(i t) \phi(-i t)-1$ is a.p. with a null generalized Fourier expansion. Accordingly, $|\phi(i t)|=1[2$, p. 27$]$.

For the reverse implication we observe that, in view of the uniqueness property of generalized Fourier expansions of a.p. functions, the relation $\phi(i t) \phi(-i t)=1$ guarantees the satisfaction of relation II.

THEOREM 2.2. Necessary and sufficient conditions that $\phi(z) \in K^{U}$ are (a) $\phi(z)$ is meromorphic, (b) $\phi(z)$ admits a Dirichlet expansion converging uniformly for $R(z) \geqq 0$, and (c) III is satisfied.

In view of Theorem 2.1 the demonstration of the present theorem may 
be patterned in an obvious way on that given for the analogous theorem with $K^{v}$ now replacing $K^{\prime}[1$, Theorem 3.2].

The following simple theorem answers a question concerning the convergence abscissa raised in the first remark on page 355 of [1].

THEOREM 2.3. If $\left\{a_{n}\right\} \in K^{U}$ (or $K^{\prime}$ ) then the uniform convergence abscissa for the associated Dirichlet series is to the left of $R(z)=0$.

We have

$$
\left|\sum a_{n} n^{-z}\right|<\epsilon, \quad R(z) \geqq 0 .
$$

In view of Lemma 2.1 and Theorem 2.1 it is easy to see that, for some positive $\delta,|\phi(s+i t)-\phi(i t)|<1 / 4$ and therefore

$$
|\phi(s+i t)|>1 / 4
$$

for all $t$ and $0 \leqq s \leqq \delta$. Accordingly, if we bear in mind Theorem 2.2, $\phi(z)$ is meromorphic and

$$
|\phi(s+i t)|<4
$$

for $-\delta \leqq s \leqq \delta$. Thus $\phi(z)$ is regular and bounded for $R(z) \geqq-\delta$, and hence by Bohr's extension of Schnee's theorem [3, p. 445] the Dirichlet series converges uniformly for $R(z)>-\delta$.

3. Basic necessity conditions. The following theorem plays a central role in the developments of this paper.

THEOREM 3.1. If $\phi(z) \in K$ then for $R(z)>0, \phi(z)$ is a regular function with a uniformly convergent Dirichlet series and $|\phi(z)| \leqq 1$.

Consider the Toeplitz matrix [4] associated with $\phi(z)$.This is defined as

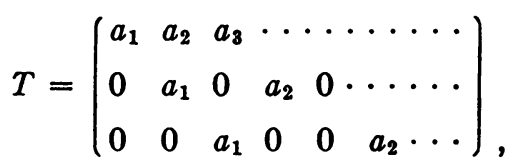

where the $n$th row has the first $n-1$ elements 0 , the $n$th is $a_{1}$, the next $n-1$ are 0 , then an $a_{2}$, then $n-1$ zeros followed by $a_{3}$, and so on. If we write $T^{\prime}$ for the transposed matrix it is clear from the relations II that $T T^{\prime}$ is the unit matrix

$$
\left(\begin{array}{llll}
1 & & & \\
& 1 & & \\
& & 1 & \\
& & & .
\end{array}\right) .
$$

Thus $T$ is a "right unitary" matrix $[5$, p. 154] and hence has the bound 1. 
According to a theorem of Toeplitz [4], this implies that $\phi(z)$ is regular and $|\phi(z)| \leqq 1$ in $R(z)>0$. Hence by the Bohr-Schnee theorem, the Dirichlet series for $\phi(z)$ converges uniformly for $R(z)>0$.

4. Uniqueness. The theorems of this section are of interest in themselves and play a role in the sequel as well.

THEOREM 4.1. If $\phi(z) \in K$ and (a) $\phi(z)$ is uniformly continuous in $R(z) \geqq 0$, (b) $|\phi(i t)|=1$, (c) $\phi(z)$ has no zeros in $R(z)>0$, and (d) $\phi(z)$ has a nonvanishing constant term in its Dirichlet expansion, then $\phi(z)= \pm 1$.

By virtue of (a) and (b),

$$
|\phi(s+i t)|>\delta \text {, }
$$

for $0 \leqq s \leqq \epsilon$. In view of Theorem $3.1, \phi(z)$ has a uniformly convergent Dirichlet expansion and is therefore a.p. for every $s>0$. Hence $|\phi(z)|$ has a positive lower bound in $0 \leqq R(z) \leqq N[2, \mathrm{p} .145]$, where $N$ may be taken arbitrarily large. If $a_{1}$ is the constant term in the expansion of $\phi(z)$, it is clear that $|\phi(z)|>\left|a_{1}\right| / 2$ for $R(z) \geqq M$. Hence $0<\rho \leqq|\phi(z)| \leqq 1, R(z) \geqq 0$.

We define an inversion by

$$
w+1=(z+1)^{-1} .
$$

The half-plane $R(z) \geqq 0$ maps into

$$
|w+1 / 2|=1 / 2 \text {. }
$$

Write $\psi(w)$ for $\phi(z(w))$. Then $u=\log |\psi(w)|$ is harmonic inside the circle of equation 4.02. Moreover, $u$ takes on the boundary value 0 a.e. Since $u$ is bounded, it follows that it is uniquely determined [6, p. 74], and is actually 0 throughout the circle of equation 4.02. Hence $|\psi(w)|=1$ or $|\phi(z)|=1$ in $R(z)>0$. It is well known that this implies $\phi(z)$ is a constant of modulus 1 $[7$, p. 120]. Hence $\phi(z)= \pm 1$.

Remark. Conditions (a) and (b) cannot be replaced by a requirement that $\phi(z)$ takes on its boundary values a.e. on $R(z)=0$.

LEMMA 4.1. If $|\psi(i t)|=1$ a.e., $\psi(z)$ is analytic in $R(z)>0$ and takes on boundary values a.e. on $R(z)=0$, and if $\infty>M>|\psi(z)|>m>0$ for $R(z)>0$, then $\psi(z)=\gamma,|\gamma|=1$.

The proof is essentially that of the last part of the demonstration of Theorem 4.1 .

The following are in a sense generalizations of Theorem 10.3 of [1]. However, they are considerably stronger and the demonstration is quite different from that of the theorem cited.

THEOREM 4.2. The functions $f^{p}(x)$ and $f^{p}(x) / C, p$ a positive integer, cannot both be numbers of $K$ unless $C=N, N$ an integer, and then $f(x)= \pm \sin N x$. 
It is understood, of course, that the inclusion $f^{p}(x) / C \in K, p$ odd, is used in the obvious sense of replacing odd function by even function in the characterization of the class $K$. Here $f^{p}(x)=d^{p} f(x) / d x^{p}$.

The case $p=1$ corresponds to Theorem 10.3 of [1] with the important generalization to functions in $K$ rather than in $K^{\prime}$. This theorem is a special case of a more general theorem which we proceed to state and prove.

Theorem 4.3. If $\left\{a_{n}\right\} \in K$ then $\left\{a_{n} n^{r} / C\right\}, r>0$, cannot be in $K$ unless $|C|=N^{r}, N$ a positive integer, and then $\left|a_{n}\right|=\delta_{n N}$.

As usual, write $\phi(z)$ for the function associated with $\left\{a_{n}\right\}$. When $\left\{a_{n} n^{r} / C\right\} \in K$ it follows from Theorem 3.1 that $\phi(z)$ has a uniformly convergent Dirichlet expansion for $R(z)>-r$. Hence $\phi(z) \in K^{U}$. In view of Theorem 2.2 we know $\phi(z)$ is meromorphic. There is no loss in generality in assuming $C$ positive. Since $\phi(z-r) / C$ is associated with $\left\{a_{n} n^{r} / C\right\}$ we have, by Theorem 3.1 ,

$$
|\phi(z-r)| \leqq C, \quad R(z)>0 .
$$

It is clear from equation 4.03 that $\phi(z)$ has no poles on $R(z)=-r$ and, in fact,

$$
|\phi(i t-r) / C| \leqq 1 \text {. }
$$

Observe now that

$$
|\phi(r-i t)|=|\phi(i t-r)|^{-1} \geqq C^{-1} .
$$

Since $\phi(z) \in K^{v}$, we infer $\phi(z)$ is uniformly continuous in $r-\epsilon \leqq R(z) \leqq r+\epsilon$, for some positive $\epsilon$. Hence, for some positive $\delta$ and $\epsilon^{\prime}$,

$$
|\phi(r-z)| \geqq \delta,
$$$$
|R(z)| \leqq \epsilon^{\prime} .
$$

Plainly equations 4.03 and 4.05 imply $|\phi(z)|$ is uniformly bounded in $R(z)>-r-\epsilon^{\prime}$. Since $\phi(z)$ is known to be meromorphic, we can assert $\phi(z)$ is actually regular in $R(z)>-r-\epsilon^{\prime}$. Hence by the Bohr-Schnee theorem the Dirichlet expansion of $\phi(z)$ surely converges uniformly for $R(z) \geqq-r$. We see now that the hypotheses of Theorem 2.2 are fulfilled by $\psi(z)=\phi(z-r) / C$ and so $\psi(z)$ satisfies relation III. Then

$$
|\phi(i t-r) / C|=1 \text {. }
$$

Let

$$
\theta(z)=C^{z / r} \phi(z)
$$

We have

$$
|\theta(z)|=1, \quad R(z)=0, R(z)=-r .
$$

The harmonic function $u=\log |\theta(z)|$ takes on the value 0 on $s=0$ and 
$s=-r$. Hence, by successive reflections first in the line $s=0$, then in the lines $s= \pm r$, then in the lines $s= \pm 2 r$, and so on, we can continue $u(s, t)$ harmonically. Thus $u=0$ for $s=0, \pm r, \pm 2 r, \cdots$. Since $\phi(z) \in K^{v}$ we know $|\phi(z)|$ has a positive lower bound in $0 \geqq R(z) \geqq-r$. Accordingly, $|u(s, t)|$ is bounded for $-r \leqq s \leqq 0$ and hence $u(s, t)$ is uniformly bounded for all values of $s$ and $t$. This implies that $|\theta(z)|$ is uniformly bounded and hence by Liouville's theorem $\theta(z)$ is a constant. Thus $\phi(z)=A C^{-z / r}$. Since $\phi(z) \in K^{v}$ it follows that $A= \pm 1$ and $C=N^{r}, N$ a positive integer.

Another result in this range of ideas follows.

ThEOREM 4.4. If $\left\{a_{n}\right\} \in K, a_{1} \neq 0$, then $\left\{n a_{n} / C \mid n=2,3, \cdots\right\}$ cannot be $a$ member of $K$ for any choice of $C$. alent,

Suppose to the contrary that $\left\{n a_{n} / C \mid n=2,3, \cdots\right\} \in K$ or, what is equiv-

$$
\frac{\phi(z-1)-a_{1}}{C} \in K
$$

We may parallel the argument used in the previous theorem to show that actually $\left\{a_{n}\right\} \in K^{U}$, that

$$
|\phi(1-i t)| \geqq\left(|C|+\left|a_{1}\right|\right)^{-1},
$$

and then that $\left(\phi(z-1)-a_{1}\right) / C$ satisfies relation III. Evidently

$$
L_{R(z) \rightarrow \infty} \phi(z)=L_{R(z) \rightarrow \infty} \phi(z-1)=a_{1} .
$$

Hence from relation III, applied to $\left(\phi(z-1)-a_{1}\right) / C$,

$$
L_{R(z) \rightarrow \infty} \phi(-z-1)=\infty \text {. }
$$

On the other hand it is plain that

$$
L_{R(z) \rightarrow \infty} \phi(-z-1)=L_{R(z) \rightarrow \infty} \phi(-z)=1 / a_{1} .
$$

This is in contradiction with the assertion of equation 4.06 , whence we gain the conclusion of the theorem.

The following theorem is a sharper form of Theorem 7.4 [1].

THEOREM 4.5. If $\left\{a_{n}^{1}\right\}$ and $\left\{a_{n}^{2}\right\}$ both belong to $U$ then it is not possible that $\left[f_{1}(m x), f_{2}(n x)\right]=0$ for all $m$ and $n$ unless either $\left\{a_{n}^{1}\right\}$ or $\left\{a_{n}^{2}\right\}$ is a null sequence.

There is no implication that either $\left\{a_{n}^{1}\right\}$ or $\left\{a_{n}^{2}\right\}$ belongs to $K^{U}$. An argument based on the a.p. property of $\phi_{1}(i t)$ and $\phi_{2}(i t)$, similar in detail to that used in the demonstration of Theorem 2.1, shows that

$$
\phi_{1}(i t) \phi_{2}(-i t)=0 \text {. }
$$

Let $L$ be a closed interval. Let $O_{1}$ and $O_{2}$ be the set of zeros in $L$ of $\phi_{1}(i t)$ and 
$\phi_{2}(i t)$ respectively. Then $O_{1} \cup O_{2}=L$. Since $L$ is of the second category [8, p. 13] there is a subinterval $L^{\prime} \subset L$ such that $O_{1}^{\prime}=L^{\prime} \cap O_{1}$ or $O_{2}^{\prime}=L^{\prime} \cap O_{2}$ is dense in $L^{\prime}$.

Suppose $O_{1}^{\prime}$ has this property. Then by continuity $\phi_{1}(i t)=0$ for all $t \in L^{\prime}$. Evidently $\sum a_{n}^{1} n^{-z}$ is continuous in a region abutting on $L^{\prime}$ on the right, for the series is obviously uniformly convergent. Hence application of the Schwartz reflection principle yields a domain of analyticity including $L^{\prime}$ in its interior. Accordingly, $\phi_{1}(z)$ vanishes in this domain and hence by analytical continuation $\phi_{1}(z)$ vanishes for $R(z)>0$. Thus since $\phi_{1}(i t)=L_{s \rightarrow 0+} \phi(s+i t)$ we have $\phi_{1}(i t)=0$ for all $t$ or $\left\{a_{n}^{1}\right\}$ is a null sequence.

We shall have use for the Fatou theorem [9] which we state in a generalized form: If $\psi(w)$ is regular and bounded in $|w|<1$ then (a) at almost all points on the circle $|w|=1, \psi(w)$ takes on boundary values $\psi\left(e^{i \theta}\right)$ for approach in the wide sense and (b) $\psi(w)$ converges in the $L_{2}$ norm topology to $\psi\left(e^{i \theta}\right)$ as $r \rightarrow 1$, that is to say

$$
L_{r \rightarrow 1} \int_{0}^{2 \pi}\left|\psi\left(r e^{i \theta}\right)-\psi\left(e^{i \theta}\right)\right|^{2} d \theta=0 .
$$

Approach in the wide sense is understood to mean approach to the boundary point exp $i \theta$ within a sector bounded by chords through this point. It is clear that a conclusion corresponding to (a) is valid for the half-plane as well. (Actually in our subsequent references we state our results for approach to the boundary along normals, but it is understood that the approach in the wide sense is permissible.) We remark also that a bounded regular function in the half plane $R(z)>0$ is uniquely determined by the boundary values approached on a set of positive measure. This is essentially an easy conclusion from a theorem of M. Riesz and F. Riesz [11]. We summarize the application of these remarks to the special case of interest to us in a theorem.

THEOREM 4.6. If $\phi(z) \in K$ then $(\mathrm{A}) \phi(s+i t)$ converges $\left({ }^{3}\right)$ a.e. to $\phi(i t)$ as $s \rightarrow 0+$, (B) $\phi(z)$ is uniquely determined by the values of $\phi(i t)$ on any set of positive measure.

5. The quasi-elementary solution. The problems raised in this section are perhaps of equal significance with the key result established in the first theorem.

Since our present definition of a quasi-elementary solution is formally different from that used in [1] we need the following lemma.

Lemma 5.1. A quasi-elementary solution belongs to $K^{U}$.

Let $\phi(z)=N(z) / D(z)$ where $N(z)$ and $D(z)$ refer to the numerator and

( $\left.{ }^{(}\right)$There is, of course, no implication that $\phi(i t)$ is the value of $\phi(z)$ for $z=i t$. Indeed in general, of course, the Dirichlet series does not converge for $R(z)=0$. 
denominator in the representation of a quasi-elementary solution. Since $D(z)$ is a.p. for each $s, s \geqq 0$, and goes to 1 uniformly as $R(z) \rightarrow \infty$ it is easy to see, just as in a similar situation treated in Theorem 4.1 , that $|D(z)| \geqq \delta>0$ for $R(z) \geqq 0$. Evidently $D(z)$ is uniformly continuous for $|R(z)| \leqq \rho, \rho>0$. Hence, for some positive $\epsilon, D(z) \geqq \delta / 2$ for $R(z) \geqq-\epsilon$. Thus $\phi(z)$ is a regular analytic function with uniformly bounded modulus in $R(z) \geqq-\epsilon$. Moreover $\phi(z)$ obviously has a convergent Dirichlet series for large enough $R(z)$. The BohrSchnee theorem guarantees that this series is surely uniformly convergent for $R(z) \geqq 0$. Since $\phi(z)$ plainly satisfies III, the proof is complete. It will appear later that actually the stronger assertion that $\phi(z) \in K^{\prime}$ is true.

THEOREM 5.1. If $\phi(z) \in K^{U}$ has a finite base, then $\phi(z)$ is a quasi-elementary solution.

We give the proof for a binary base, $\left\{p_{i} \mid i=1,2\right\}$. The extension to a general finite base will be obvious. Let

$$
w_{i}=p_{i}^{-2},
$$

Then

$$
\phi(z)=\sum a(k, l)\left(p_{1}^{k} p_{2}^{l}\right)^{-z}
$$

becomes

$$
F\left(w_{1}, w_{2}\right)=\sum a(k, l) w_{1}^{k} w_{2}^{l},
$$

where we write $a(k, l)$ instead of $a_{n}, n=p_{1}^{k} p_{1}^{l}$.

In view of Theorem 2.3 the series for $\phi(z)$ converges for $z=-\gamma<0$. Hence the series for $F\left(w_{1}, w_{2}\right)$ converges for $w_{i}=p_{i}^{-\gamma}$. Accordingly the latter series is absolutely convergent and defines an analytic function $[11$, p. 36] for $\left|w_{i}\right| \leqq 1+\epsilon_{0}<p_{i}^{\gamma}$.

Incidentally, the simple argument above shows that $K^{v}$ and $K^{\prime}$ are identical for solutions with a finite base. Let $S(\phi)$ be the range of $\phi(z)$ for $R(z)=0$. Similarly, $S(F)$ is the range of $F\left(w_{1}, w_{2}\right)$ for $\left|w_{1}\right|=1,\left|w_{2}\right|=1$. According to a result of Bohr's [3, p. 448], $S(F)$ is the closure of $S(\phi)$. Since the complex numbers in $S(\phi)$ have absolute value 1 , it follows that the complex numbers in $S(F)$ have absolute value 1 also.

We wish to show that in the region $\Delta$, defined by

$$
\left|w_{1}\right|=\overrightarrow{p_{1}}, \quad\left|w_{2}\right|=\overrightarrow{p_{2}}, \quad\left(1+\epsilon_{0}\right)^{-1}<\overrightarrow{p_{2}}<1+\epsilon_{0},
$$

we have

$$
F\left(w_{1}, w_{2}\right)=\left(F\left(w_{1}^{-1}, w_{2}^{-1}\right)\right)^{-1} .
$$

For this purpose we need to extend the argument given by Bohr for a previously quoted theorem $[3, \mathrm{p} .448]$. We remark first that in view of uniform 
convergence we can choose $N$ so large that, for $R(z)$ consistent with equation 5.02,

$$
\left|\sum_{N+1}^{\infty} a_{n} n^{-z}\right|<\delta, \quad \delta>0, n=p_{1}^{k} p_{2}^{l}
$$

Similarly

$$
\left|\sum_{N+1}^{\infty} a(l, k) w_{1}^{k} w_{2}^{2}\right|<\delta
$$

for $\left|w_{i}\right|=p_{i}^{-s}$ or $\left|w_{i}\right|=p_{i}^{s}$ in $\Delta$. Let $w_{1}=p_{1}^{-s+i t}, w_{2}=p_{2}^{-s+i r}$. Write $\mu$ for log $p_{1}$ and $\nu$ for $\log p_{2}$. We define $F_{N}\left(w_{1}, w_{2}\right)$ and $F_{N}^{\prime}\left(w_{1}, w_{2}\right)$ by

$$
\begin{aligned}
& F_{N}\left(w_{1}, w_{2}\right)=\sum_{k, l=1}^{N} a(k, l) w_{1}^{l} w_{2}^{k}=\sum_{1}^{N} a(k, l)\left(\overrightarrow{p_{1}} e^{i t \mu}\right)^{k}\left(\vec{p}_{2}^{-k} e^{i r \eta}\right)^{l}, \\
& F_{N}^{\prime}\left(w_{1}, w_{2}\right)=\sum_{k, l=1}^{N} a(k, l)\left(\overrightarrow{p_{1}} e^{i t \mu}\right)^{-k}\left(\overrightarrow{p_{2}} e^{i r r}\right)^{-l} .
\end{aligned}
$$

We define $\phi_{N}(z)$ and $\phi_{N}^{\prime}(z)$ by replacing $t$ and $r$ by the single variable $h$ in $F_{N}$ and $F_{N}^{\prime}$ respectively. By the classical Kronecker theorem on diophantine approximation, for each positive number $\delta^{\prime}$ we can choose $h$ so that for some integers $a, b,|(t-h) \mu+2 \pi a|<\delta^{\prime} / N,|(r-h) \nu+2 \pi b|<\delta^{\prime} / N$. Accordingly

$$
\begin{aligned}
\left|\phi_{N}(z)-F_{N}\left(w_{1}, w_{2}\right)\right| & \leqq \sum_{1}^{N}|a(k, l)|\left(p_{1}^{-k} p_{2}^{-l}\right)^{\prime}\left|e^{i k(\mu k+\nu l)}-e^{i(\mu k t+\nu l r)}\right| \\
& \leqq 2 \delta^{\prime} \sum_{1}^{N}|a(k, l)|\left(p_{1}^{k} p_{2}^{l}\right)^{-*} .
\end{aligned}
$$

Similarly

$$
\left|\phi_{N}^{\prime}(z)-F_{N}^{\prime}\left(w_{1}, w_{2}\right)\right| \leqq 2 \delta^{\prime} \sum_{1}^{N}|a(k, l)|\left(p_{1}^{k} p_{2}^{l}\right)^{\prime} .
$$

Choose $\delta^{\prime}$ such that the right-hand side of equation 5.05 , and hence the righthand side of equation 5.04 also, is inferior to $\delta$. Hence, since $\phi(z) \phi(-z)=1$, we have for the case characterized in equation $\mathbf{5 . 0 2}$

$$
\begin{aligned}
\mid F\left(w_{1}, w_{2}\right)-( & \left.F\left(w_{1}^{-1}, w_{2}^{-1}\right)\right)^{-1} \mid \\
& \leqq\left|F\left(w_{1}, w_{2}\right)-\phi(z)\right|+\mid(\phi(-z))^{-1}-\left(F\left(w_{1}^{-1}, w_{2}^{-1}\right)^{-1} \mid\right. \\
& \leqq\left|F_{N}\left(w_{1}, w_{2}\right)-\phi_{N}(z)\right|+\left|F_{N}^{\prime}\left(w_{1}, w_{2}\right)-\phi_{N}^{\prime}(z)\right|+4 \delta \\
& \leqq 6 \delta .
\end{aligned}
$$

Since $\delta$ is arbitrary, it is clear that we have established the assertion in equation 5.03 under the conditions of equation 5.02. 
Consider $F\left(w_{1}, c\right)$ with $c$ fixed, where $c=p_{2}{ }^{s+i \alpha},\left(1+\epsilon_{0}\right)^{-1}<|c|<1+\epsilon_{0}$. We assert that $F\left(w_{1}, c\right)$ is a rational function of $w$. We remark first that $F\left(w_{1}, c\right)$ may be continued by means of the relation

$$
F\left(w_{1}, c\right)=\left(F\left(w_{1}^{-1}, c^{-1}\right)\right)^{-1} \text {. }
$$

Indeed, for $\left|w_{1}\right|=p_{1}^{s}$, equation 5.06 is a consequence of equation 5.03. Then since the left-hand side of equation 5.06 is analytic in $\left(1+\epsilon_{0}\right)^{-1}<\left|w_{1}\right|$ $<\left(1+\epsilon_{0}\right)$, this equation holds for $\left|w_{1}\right|=1$. On making use of an extension of the Schwarz reflection theorem $[7$, p. 157] one can demonstrate precisely as in a somewhat simpler situation treated previously (Lemma 3.1 of [1]) that an analytic continuation is indeed defined by equation 5.06. Suppose now that $w_{1}^{\prime}$ is a singular point. Plainly $\left|w_{1}^{\prime}\right|>1$ since we have already shown that $F\left(w_{1}, w_{2}\right)$ is analytic for $\left|w_{i}\right|<1+\epsilon_{0}$. According to equation 5.06, if the singularity were nonpolar, then $F\left(w_{1}, c^{-1}\right)$ would have a singularity for $w_{1}=\left(w_{1}^{\prime}\right)^{-1}$. However, under the restriction on $c$ and $w_{1}^{\prime}, F\left(w_{1}, c\right)$ is analytic at $\left(w_{1}^{\prime}\right)^{-1}$. Hence any singularities of $F\left(w_{1}, c\right)$ in the extended plane must be poles, and $F\left(w_{1}, c\right)$ is a rational function.

In a similar manner we can show that $F\left(d, w_{2}\right)$ is a rational function of $w_{2}$ when $d$ is restricted to some ring containing $\left|w_{1}\right|=1$ in its interior. We recapitulate the features significant for our purpose. For some positive $\epsilon^{\prime}$ and with $D_{1}$ and $D_{2}$ referring to the domains $\left|w_{1}-1\right|<\epsilon^{\prime}$ and $\left|w_{2}^{\prime}-1\right|<\epsilon^{\prime}$ respectively, $F\left(w_{1}, w_{2}\right)$ is analytic for $w_{1} \in D_{1}, w_{2} \in D_{2}$. Moreover $F\left(a_{1}, w_{2}\right)$ is a rational function of $w_{2}$ for $a_{1} \in D_{1}$ and $F\left(w_{1}, a_{2}\right)$ is a rational function of $w_{1}$ for $a_{2} \in D_{2}$. These facts ensure that $F\left(w_{1}, w_{2}\right)$ is a rational function of both variables $[11$, Theorem 2 , p. 238]. Therefore

$$
F\left(w_{1}, w_{2}\right)=B\left(w_{1}, w_{2}\right) / C\left(w_{1}, w_{2}\right),
$$

where $B\left(w_{1}, w_{2}\right)$ and $C\left(w_{1}, w_{2}\right)$ are relatively prime polynomials. An important property of these polynomials for the last stage of the proof is that, for $\left|w_{i}\right|<1+\epsilon_{0}$, the zero curves of $B\left(w_{1}, w_{2}\right)$ and $C\left(w_{1}, w_{2}\right)$ are disjunct. This is a consequence of the analyticity of $F\left(w_{1}, w_{2}\right)$ in the domain cited, cf., for instance, the theorem of Weierstrass [11, p. 197]. We infer then that $\left|C\left(w_{1}, w_{2}\right)\right|$ is bounded from below by a positive constant in this domain.

It is now clear that equation 5.03 extends to all values of $w_{1}$ and $w_{2}$ by analytic continuation. (It is understood, of course, that at possible singularities this equation is to be interpreted in the sense of equality of limiting values along curves in correspondence through the generalized inversion with respect to the origin, namely $w_{1}$ and $w_{2}$ replaced by $w_{1}^{-1}$ and $w_{2}^{-1}$.)

We observe that $F\left(w_{1}, w_{2}\right)$ has no nonessential singularity of the second kind at $\left(w_{1}^{\prime}, w_{2}^{\prime}\right),\left|w_{i}^{\prime}\right|>\left(1+\epsilon_{0}\right)^{-1}$. Thus $B\left(w_{1}, w_{2}\right)$ and $C\left(w_{1}, w_{2}\right)$ have no common zeros in the domains $Q_{1}:\left|w_{i}\right|<1+\epsilon_{0}$ and $Q_{2}:\left|w_{i}\right|>\left(1+\epsilon_{0}\right)^{-1}$.

On turning to $\phi(z)$ it is clear that we have shown that

$$
\phi(z)=B(z) / C(z)
$$


where

$$
\begin{aligned}
& B(z)=\sum b(j, k)\left(p_{1}^{j} p_{2}^{k}\right)^{-z}, \\
& C(z)=\sum c(l, r)\left(p_{1}^{l} p_{2}^{r}\right)^{-z},
\end{aligned}
$$

and both sums are finite with $B(z)$ and $C(z)$ relatively prime. We can dispose of the case $C(z)$ constant, immediately. Indeed, Theorem 2.2 of [1] shows that $B(z)$ must perforce be a constant also. If $B(z)$ is constant, then III implies

$$
\sum c(l, r)\left(p_{1}^{l} p_{2}^{r}\right)^{z}=1 / C(z)=\sum_{m=1}^{\infty} g_{m} m^{-z}, \quad|R(z)| \leqq \epsilon .
$$

The uniqueness of Dirichlet expansions guarantees then that $C(z)$ is a constant also. Hence if either $B(z)$ or $C(z)$ is constant, $\phi(z)= \pm 1$ and our theorem is established. We may therefore assume that neither $B(z)$ nor $C(z)$ is constant in the sequel.

In view of relation III

$$
B(z) / C(-z)=C(z) / B(-z)=q(z) .
$$

Evidently $q(z)$ is single-valued. We observe that the range of $B(z)$ or $C(z)$ for $R(z)=s$ is dense in the range of $B\left(w_{1}, w_{2}\right)$ or $C\left(w_{1}, w_{2}\right)$ respectively for $\left|w_{i}\right|=p_{i}^{-i}$. In view of the italicized statement above, it follows that $B(z)$ and $C(z)$ have no common zeros. Indeed $Q_{1}$ takes care of $s \leqq 0$ and $Q_{2}$ accounts for $s>0$. It is impossible that $B(z)$ have a zero in the left half-plane $R(z)<0$. Otherwise, since

$$
|\phi(z)|=|C(-z) / B(-z)| \leqq 1, \quad R(z) \geqq 0,
$$

we should have $C(z)=0$ for the same value of $z$. The possible zeros of $q(z)$ are, according to equation 5.10 , common to $B(z)$ and $C(z)$. Hence $q(z)$ is zero-free in $R(z)>-\epsilon$. The possible poles of $q(z)$ are at points for which $B(-z)=0$. Hence $q(z)$ has no poles for $R(z)>-\epsilon$.

Since $\phi(z) \in K^{\prime}$, we know there are no zeros in $|R(z)| \leqq \epsilon^{\prime}<\rho, \epsilon^{\prime}>0$. Hence $B(z)$ does not vanish in $R(z) \leqq \epsilon^{\prime}$. Since

$$
L_{R(z) \rightarrow-\infty} B(z)=\infty
$$

it is easy to show that $|B(z)|$ has a positive lower bound for $R(z) \leqq \epsilon^{\prime}$. The argument is precisely that used in the first part of Theorem 4.1. Similarly we can show that $|C(z)|$ has a positive lower bound in $R(z) \geqq-\epsilon^{\prime}$.

We now make the important observation that $c(0,0)=0$. If not, $F\left(w_{1}, w_{2}\right)$ has a nonessential singularity of either the first (polar) or second kind at $(0,0)$. Neither alternative is admissible.

Let $M=p_{1}^{a} p_{2}^{b}$ be the term of "highest degree" in $B(z)$ in the sense that $p_{1}^{a} p_{2}^{b}>p_{1}^{j} p_{2}^{k}$ for $(j, k) \neq(a, b)$, where comparison is made for all terms in $B(z)$ with nonvanishing $b(k, l)$ 's. Then 


$$
B(-z)=M^{z}\left(d+\sum_{j+k>0} d(j, k)\left(p_{1}^{j} p_{2}^{k} / M\right)^{z}\right) .
$$

Hence

$$
q(z) M^{z}=\left(c+\sum_{l+r>0} C(l, r)\left(p_{1}^{l} p_{2}^{r}\right)^{-z}\right) /\left(d+\sum_{j+k>0} d(j, k)\left(p_{1}^{j} p_{2}^{k} / M\right)^{-z}\right) .
$$

We now make use of the inferences concerning the positive lower bounds of $|B(-z)|$ and $|C(z)|$ in $-\epsilon^{\prime} \leqq R(z)$. Thus

$$
L_{R(z) \rightarrow \infty} q(z) M^{z}=c / d \neq 0,
$$

and then

$$
0<h \leqq\left|q(z) M^{z}\right| \leqq H<\infty,
$$$$
R(z) \geqq-\epsilon^{\prime} .
$$

We observe that $q(z)$ satisfies III and is analytic for $R(z) \geqq-\epsilon^{\prime}$. It is then apparent, in view of equation 5.11, that the hypotheses of Lemma 4.1 are satisfied. Accordingly, there is a constant $L$ such that

$$
q(z) M^{z}=L, \quad|L|=1 .
$$

This is to say,

$$
B(z)=L M^{-z} C(-z) .
$$

Since $\phi(z)$ is real for real $z$-values, we conclude that the coefficients $c(l, r)$ are real and $L= \pm 1$. Thus

$$
\phi(z)= \pm M^{-z}\left(1+\sum_{l+r>0} c(l, r)\left(p_{1}^{l} p_{2}^{r}\right)^{z}\right) /\left(1+\sum_{l+r>0} c(l, r)\left(p_{1}^{l} p_{2}^{r}\right)^{-z}\right) .
$$

The denominator has a modulus bounded from below in $R(z) \geqq-\epsilon^{\prime}$. Moreover it is manifest that $M$ must be a multiple of the L.C.M. of $\left\{p_{1}^{l} p_{2}^{r}\right\}$. Hence $\phi(z)$ is a quasi-elementary solution and our proof is complete.

Theorem 5.2. If $\phi(z) \in K^{\prime}$ has the base $\left\{p_{n} \mid n=1, \cdots\right\}$ then the Dirichlet series $\psi(z)$, obtained by replacing $p_{n}$ by $p_{n}^{\prime}$ where $\left\{p_{n}^{\prime}\right\}$ is a set of not necessarily distinct primes, belongs to $K^{\prime}$ also.

Thus suppose

$$
\phi(z)=\sum a\left(l_{1}, \cdots, l_{j}\right)\left(p_{1}^{l_{1}} \cdots p_{j}^{l_{j}}\right)^{-z}
$$

has the base $\left\{p_{n}\right\}$. Replace $p_{i}^{-3}$ by $w_{i}$ in equation 5.12. The resulting function is denoted by $F\left\{w_{n}\right\}$. For $R(z)=0$ the range of $\phi(z)$ is dense in the range of $F\left\{w_{n}\right\}$ for $\left|w_{n}\right|=1$. Since $\phi(z) \in K^{\prime}$ it follows just as in the first part of the proof of the previous theorem that the range of $F\left\{w_{n}\right\}$ for $\left|w_{n}\right|=1$ is $a s u b$ set of the unit circle in the complex plane. The arguments of the various $w_{n}$ 's can be given arbitrary values mod $2 \pi$. Hence, for instance, taking the argu- 
ment of $w_{n}$ as $\log p_{n}{ }^{\prime} t$ rather than $\log p_{n} t$ (where $p_{n}^{\prime}=1$ is included) does not affect the italicized property of the range set of $F\left\{w_{n}\right\}$. This is readily seen to imply the assertion of the theorem. In particular, every reduction of a $\phi(z) \in K^{\prime}$ to a function on a finite base by identifying a suitable number of $p_{n}$ 's leads to a quasi-elementary solution.

Remark. In view of the last two theorems it is natural to raise the question : If $\phi(z) \in K^{\prime}$ does $\phi(z)$ necessarily have a finite base? A direct attack following the lines of Theorem 5.1 meets difficulties which constitute interesting problems in the theory of analytic functions of an infinite number of complex variables. Thus does a rational function $F\left\{w_{n}\right\}$ permit the representation $C\left\{w_{n}\right\} F\left\{w_{n}\right\}=B\left\{w_{n}\right\}$ where $B\left\{w_{n}\right\}$ and $C\left\{w_{n}\right\}$ are entire functions whose finite sections $\left(w_{n}=0, n \geqq N\right)$ or $\left(w_{n}=1, n \geqq N\right)$ are polynomials? Does a meromorphic function permit a representation of this sort where $B\left\{w_{n}\right\}$ and $C\left\{w_{n}\right\}$ are relatively prime entire functions? This is a generalization of Poincaré's problem for functions of a finite number of complex variables. The representation of the general solution on a finite base, of the type of equation 10.07 for the one-element base, is another problem of prime importance. It is closely related to a representation problem for functions of several complex variables which take on boundary values of constant modulus on the (distinguished) surface $\left\{\left|w_{i}\right|=1\right\}$. The writer will present results in this direction in a later communication.

THEOREM 5.3. If (a) $\phi(z)=A(z) / B(z) \in K^{\prime}$, (b) $A(z), B(z)$ are entire functions with no common zeros in $R(z) \geqq 0$, (c) $B(z)$ has a Dirichlet expansion converging uniformly for $R(z) \geqq 0$, (d) $\left|M^{2} A(z) / B(-z)\right| \geqq h>0$ for $R(z) \geqq 0$, then $\phi(z)$ is a quasi-elementary solution.

In view of relation III it is easy to see that

$$
\mu(z)=A(z) / B(-z)=B(z) / A(-z)
$$

and

$$
\mu(z) \mu(-z)=1 .
$$

Evidently $B(z)$ is zero-free for $R(z) \geqq 0$ in view of conditions (a) and (b). Hence $\mu(z)$ is regular and zero-free in $R(z) \geqq 0$. It is clear that the hypotheses of Lemma 4.1 are satisfied by $M^{z} \mu(z)$. Accordingly

$$
\mu(z)=L M^{-z} \text {, }
$$$$
|L|=1 \text {. }
$$

Hence

$$
\phi(z)=L M^{-z} B(-z) / B(z) .
$$

Evidently $(B(z))^{-1}$ and $B(-z)$ are a.p. for $R(z)=0$. If the expansion for $B(z)$ were not finite, then $L M^{-i t} B(-i t)$ would have terms in its expansion with positive imaginary parts for some of the exponents. This would be true then 
of $\phi(i t)$, also. Such a contingency is barred by the fact that $\phi(z) \in K^{\prime}$. Hence $B(z)$ has a finite expansion. The rest of the demonstration is obvious. It will be noted that in particular we have shown that no $\phi(z)$ with an infinite basis can satisfy the condition of the theorem.

6. Dirichlet series conditions. Our relations I and II are necessary and sufficient conditions in terms of $\{f(n x)\}$ and $\left\{a_{n}\right\}$ for a solution in $K$. Relation III is an analogous condition under the restriction $\phi(z) \in K^{U}$. The possibility that relation III is actually effective for the general case is disposed of by the examples of a later section. Nevertheless we are able to give necessary and sufficient conditions in terms of the properties of the Dirichlet series and thus complete our picture in an essential way. This is the contribution of the theorem below.

THEOREM 6.1. Necessary and sufficient conditions in order that $\phi(z) \in K$ are (a) $|\phi(z)| \leqq 1, R(z)>0$, (b) $\phi(z)$ has a uniformly convergent Dirichlet series for $R(z)>0$ or $\left(\mathrm{b}^{\prime}\right) \phi(z)$ has a Dirichlet expansion and is regular for $R(z)>0$, (c) $\sum a_{n}^{2}=1$ or $\left(c^{\prime}\right) L_{s \rightarrow 0+} M\{\phi(z)\}=1$.

We prove the theorem under conditions (a), (b), (c). The possibilities $\left(b^{\prime}\right)$ or $\left(c^{\prime}\right)$ are easily shown to be equivalent to $(b)$ or $(c)$. That the conditions are necessary is plain in view of Theorem 3.1. We need therefore consider the sufficiency aspect alone.

The boundedness of $|\phi(z)|$ guarantees the existence of the following integral means:

$$
M\left\{|\phi(z)|^{2}-1\right\}=1-2 M\{\phi(z)\}+M\left\{|\phi(z)|^{2}\right\}, \quad R(z)>0 .
$$

According to a well known theorem [7, p. 307] we have

$$
1=\sum a_{n}^{2}=L_{s \rightarrow 0+} M\{\phi(z)\} .
$$

The left-hand side of equation 6.01 is obviously non-negative. Hence, using equation 6.02 , we get

$$
\begin{aligned}
& -1+\liminf _{s \rightarrow 0+} M\left\{|\phi(z)|^{2}\right\} \geqq 0, \\
& -1+\underset{s \rightarrow 0+}{\lim \sup } M\left\{|\phi(z)|^{2}\right\} \geqq 0 .
\end{aligned}
$$

Accordingly, in view of condition (a), the indicated limit inferior and limit superior are equal and

$$
L_{8 \rightarrow 0+} M\left\{|\phi(z)|^{2}\right\}=1 .
$$

Since $\phi(z)$ is an a.p. function for all $s>0$, so is $|\phi(z)|^{2}$. Hence

$$
L_{s \rightarrow 0+} \sum_{(k, l)=1}\left(A_{k, l}(s)\right)^{2}=L_{s \rightarrow 0+} M\left\{|\phi(z)|^{2}\right\}=1,
$$


where

$$
A_{k, l}(s)=\sum_{j=1}^{\infty} a_{k j} a_{l j}\left(j^{2} k l\right)^{-s}
$$

We have

$$
\left|\sum a_{n}^{2}-\sum a_{n}^{2} n^{-28}\right| \leqq \sum_{n=1}^{N} a_{n}^{2}\left(1-n^{-28}\right)+2 \sum_{n=N+1}^{\infty} a_{n}^{2}, \quad s \geqq 0 .
$$

For arbitrary $\epsilon, \epsilon>0$, we can choose $N$ and a positive $\delta$ such that both sets of terms on the right-hand side of equation 6.05 are inferior to $\epsilon / 2$ for $0 \leqq s<\delta$. Hence

$$
L_{s \rightarrow 0+} A_{1,1}(s)=\sum a_{n}^{2}=A_{1,1}(0)=1 .
$$

For $\rho>0$, we choose a positive $\mu$ such that

$$
\left(A_{1,1}(s)\right)^{2} \geqq 1-\rho
$$

for all $0<s \leqq \mu$. In view of equation 6.03 we have, then,

$$
\sum_{(k, l)=1, k l \neq 1}\left(A_{k, l}(s)\right)^{2}<\rho .
$$

Accordingly

$$
L_{s \rightarrow 0+}\left|A_{k, l}(s)\right|=0, \quad(k, l)=1, k l \neq 1 .
$$

An obvious application of the Schwarz inequality shows that $A_{k, l}(0)$ exists and that its series representation (equation 6.04) converges absolutely. A simple argument then ensures, in view of equation 6.07 and the Abel theorem for Dirichlet series [7, p. 291], that

$$
A_{k, l}(0)=\sum_{j=1}^{\infty} a_{j k} a_{j l}=0, \quad(k, l)=1, k l \neq 1 .
$$

Since equations 6.06 and 6.08 assert that relation II is satisfied, our proof is complete.

7. Unitary transformations. In $\$ 3$ we showed that the sequence $\left\{a_{n}\right\} \in K$ determines a transformation which is unitary on the right, although except for the trivial case $a_{n}= \pm \delta_{1 n}$ the transformation is not unitary. This result suggests the possibility of utilizing the sequence $\left\{a_{n}\right\}$ in other ways to define a transformation which is actually unitary. We show below that in a fairly general case, at least, this can be done.

Our first theorem yields another necessary condition for $\phi(z) \in K$. In the course of the proof we introduce a number of concepts that have later application, and this makes the developments considerably longer than necessary for this theorem alone. 
THEOREM 7.1. If $\phi(z) \in K$ then the function $\left({ }^{3}\right) \phi(i t)$ defined in Theorem 4.6 satisfies

$$
L_{s \rightarrow 0+} \int_{-\infty}^{\infty}\left|\frac{\phi(s+i t)}{1 / 2-s-i t}-\frac{\phi(i t)}{1 / 2-i t}\right|^{2} d t=0 .
$$

Write

$$
H(\lambda)=\sum_{n>1 / \lambda} a_{n} n^{-1 / 2}
$$

The following relations are valid for a range of $s$ values to be specified presently:

$$
\begin{gathered}
Q(\lambda, s)=\lim _{T \rightarrow \infty} \frac{1}{2 \pi} \int_{-T}^{T} \frac{\phi(s+i t)}{1 / 2-s-i t} \lambda^{-(s+1 / 2+i t)} d t, \\
\phi(s+i t) / 2^{-1}-s-i t=\underset{A \rightarrow \infty}{\lim .} \int_{A^{-1}}^{A} Q(\lambda, s) \lambda^{i t-1 / 2} d \lambda .
\end{gathered}
$$

We refer to $Q(s, \lambda)$ and $\phi(s+i t) / 2^{-1}-s-i t$ as $M$ transforms. The term is used to indicate the connection with the Mellin transform where, however, $Q$ would be free of $s$. Our formulation preserves the relation to Fourier transforms.

The spaces $L_{2}(0, \infty)$ and $L_{2}(-\infty, \infty)$ are equivalent [8, p. 180], and if $E$ is the linear homeomorphism connecting them, we write with an obvious interpretation

$$
\begin{aligned}
E L_{2}(0, \infty) & =L_{2}(-\infty, \infty), \\
E^{-1} L_{2}(-\infty, \infty) & =L_{2}(0, \infty) .
\end{aligned}
$$

We say the linear operator $P$ on $L_{2}(-\infty, \infty)$ to $L_{2}(0, \infty)$ is essentially unitary if $E P$ is unitary. Similarly, the linear operator $P^{\prime}$ on $L_{2}(0, \infty)$ to $L_{2}(-\infty, \infty)$ is essentially unitary if $E^{-1} P^{\prime}$ is unitary. The operator taking a function into its $M$ transform is essentially unitary. In particular,

$$
\int_{0}^{\infty}(Q(s, \lambda))^{2} d \lambda=\frac{1}{2 \pi} \int_{-\infty}^{\infty}\left|\frac{\phi(s+i t)}{1 / 2-s-i t}\right|^{2} d t .
$$

Since $\phi(z) \in K$ it follows from Theorem 3.1 that

$$
\phi(s+i t) / 2^{-1}-s-i t \in L_{2}(-\infty, \infty), \quad 0<s<1 / 2 .
$$

Hence equations 7.02 and 7.03 are valid for $0<s<1 / 2$ and $Q(s, \lambda) \in L_{2}(0, \infty)$. Write

$$
\phi_{N}(z)=\sum_{n=1}^{N} a_{n} n^{-z} .
$$

Then the $\mathrm{M}$ transform of $\phi_{N}(s+i t) / 2^{-1}-s-i t$ exists as a function in 
$L_{2}(0, \infty)$ for $0<s<1 / 2$ and may be denoted by $Q_{N}(s, \lambda)$. It is easy to verify that

$$
Q_{N}(s, \lambda)=\lambda^{\star-1} \sum_{n>1 / \lambda}^{N} a_{n} n^{-1 / 2}
$$

We have

$$
\begin{aligned}
& \int_{-\infty}^{\infty}\left|\frac{\phi(s+i t)-\phi_{N}(s+i t)}{1 / 2-s-i t}\right|^{2} d t \\
&=\int_{-\infty}^{\infty}\left|\sum_{n=N+1}^{\infty} a_{n} n^{-s-i t}\right|^{2} /\left((1 / 2-s)^{2}+t^{2}\right) \cdot d t .
\end{aligned}
$$

According to Theorem 3.1, the Dirichlet expansion for $\phi(z)$ is uniformly convergent for $s>0$. Hence the limit as $N \rightarrow \infty$ of the left-hand side of equation 7.06 is 0 . That is to say, $\phi_{N}(s+i t) / 2^{-1}-s-i t$ converges to $\phi(s+i t) / 2^{-1}-s-i t$ in the norm topology of $L_{2}(-\infty, \infty)$. Since an essentially unitary operator is plainly continuous in the norm topology, it follows that $Q_{N}(\lambda, s)$ converges to $Q(\lambda, s)$ in the norm topology of $L_{2}(0, \infty)$. However, $Q_{N}(s, \lambda)$ obviously converges in the usual sense to $H(\lambda) \lambda^{-1-1}$ as $N \rightarrow \infty$. We make use of the principle that a mean $L_{2}$ limit and an ordinary limit must be essentially the same, and infer $Q(s, \lambda)=H(\lambda) \lambda^{--1}$ a.e.

Observe now that evidently

$$
L_{s \rightarrow 0+} H(\lambda) \lambda^{\circ-1}=H(\lambda) / \lambda .
$$

We combine Fatou's lemma $[7$, p. 346] and equation 7.05 to get

$$
\begin{aligned}
\int_{0}^{\infty}\left(H(\lambda) \lambda^{-1}\right)^{2} d \lambda & \leqq \liminf _{s \rightarrow 0+} \int_{0}^{\infty}\left|H(\lambda) \lambda^{-1}\right|^{2} d \lambda \\
& \leqq \liminf _{s \rightarrow 0+} \int_{0}^{\infty}\left|\frac{\phi(s+i t)}{1 / 2-s-i t}\right|^{2} d t / 2 \pi \\
& \leqq \liminf _{s \rightarrow 0+} \int_{0}^{\infty}\left|\frac{1}{1 / 2-s-i t}\right|^{2} d t / 2 \pi \\
& \leqq 1 .
\end{aligned}
$$

Hence $H(\lambda) / \lambda \in L_{2}(0, \infty)$. Obviously

$$
\int_{0}^{\infty}\left(\frac{B(\lambda)}{\lambda}\right)^{2}\left(\lambda^{s}-1\right)^{2} d \lambda=\int_{0}^{\infty}\left(H\left(\mu^{-1}\right)\left(\mu^{-s}-1\right)\right)^{2} d \mu .
$$

Choose $A, A>1$, so large that $\int_{\Lambda}^{\infty}\left(H\left(\mu^{-1}\right)\right)^{2} d \mu<\epsilon$. Thus

$$
\int_{A}^{\infty}\left(H\left(\mu^{-1}\right)\left(\mu^{-s}-1\right)\right)^{2} d \mu<2 \epsilon \text {. }
$$


We have

$$
\int_{0}^{A}\left(H\left(\mu^{-1}\right)\left(\mu^{-s}-1\right)\right)^{2} d \mu \leqq B \int_{0}^{A}\left(\mu^{-s}-1\right)^{2} d \mu
$$

Plainly, for all positive sufficiently small values of $s$, the right-hand side of equation 7.09 is inferior in value to $\epsilon$. Hence the left-hand side of equation 7.08 goes to 0 with $s \rightarrow 0+$. That is to say, $H(\lambda) \lambda^{8-1}$ converges to $H(\lambda) \lambda^{-1}$ in the norm topology of $L_{2}(0, \infty)$ as $s \rightarrow 0+$. If we make use of the fact that we are dealing with essentially unitary operators, it is then clear that $\phi(s+i t) / 2^{-1}-s-i t$ must converge to a function $\psi(i t)$ in the norm topology of $L_{2}(-\infty, \infty)$ and, moreover, $\psi(i t)$ is the $\mathrm{M}$ transform of $H(\lambda) \lambda^{-1}$.

According to Theorem $4.6, \phi(s+i t) / 2^{-1}-s-i t$ converges a.e. in the usual sense to $\phi(i t) / 2^{-1}-i t$ as $s \rightarrow 0+$. Hence, again making use of the identity of $L_{2}$ norm and ordinary limits, we conclude that $\psi(i t)=\phi(i t) / 2^{-1}-i t$ a.e.

ThEOREM 7.2. If $\phi(z) \in K$ and if (a) the function $\phi(i t)$ shown to exist in Theorem 4.6 has its modulus 1 almost everywhere, then

$$
H(\lambda) \lambda^{-1} \in L_{2}(0, \infty)
$$

$$
\int_{0}^{\infty}\left(H(\lambda) \lambda^{-1}\right)^{2} d \lambda=1
$$

$$
\int_{0}^{\max \left(k^{-1}, \Gamma^{-1}\right)} \frac{H(k \lambda) H(l \lambda)}{\lambda^{2}} d \lambda=\min (k, l)\left(1-(\phi(1 / 2))^{2}\right),
$$

(D) $H(\lambda \rho) / \lambda$ is a self-reciprocal kernel defining a unitary transformation in $L_{2}(0, \infty)$.

Conclusions (A) and (B) follow directly from Theorem 7.1, equation 7.05, and condition (a). The fact that $|\phi(i t)|=1$ a.e. and that $H(\lambda) \lambda^{-1}$ is the $M$ transform of $\psi(i t)$ guarantee [12] that

$$
\int_{0}^{\infty} \frac{H(k \lambda) H(l \lambda)}{\lambda^{2}} d \lambda=\min (k, l), \quad k>0, l>0 .
$$

The definition of $H(\lambda)$ makes clear that $H(\lambda)=\phi(1 / 2)$ for $\lambda>1$. Thus the left-hand side of equation 7.10 is

$$
\int_{0}^{\max \left(k^{-1}, l^{-1}\right)} \frac{H(k \lambda) H(l \lambda)}{\lambda^{2}} d \lambda+\phi(1 / 2)^{2} \int_{\max \left(k^{-1}, r^{-1}\right)}^{\infty} d \lambda / \lambda^{2}
$$

Therefore

$$
\int_{0}^{\max \left(x^{-1}, \Gamma^{-1}\right)} \frac{H(k \lambda) H(l \lambda)}{\lambda^{2}} d \lambda=\min (k, l)\left(1-(\phi(1 / 2))^{2}\right) .
$$


The relation in equation 7.11 is referred to by the letter $\mathrm{W}$ in the sequel. Conclusion (D) is a consequence of (C) [12].

TheOREM 7.3. If (a) $\phi(z) \in K^{U}$ or (b) $\phi(z) \in K$, and $\phi(z)$ has a one-element base, then the conclusions of the previous theorem are valid.

In case (a) it is evident that $\phi(i t)$ is the value of $\phi(z)$ for $z=i t$. Hence $|\phi(i t)|=1$ by Theorem 2.2. For case (b) we refer to Theorem 10.3 of $\S 10$, where it is shown that $|\phi(i t)|=1$.

Remark. In view of Theorem 7.3 it seems highly desirable to complete our theory by deciding whether condition (a) in Theorem 7.2 is necessary. The situation may perhaps be clarified by the following observation. If we make use of relation II it is possible to show in a fairly straightforward manner that, formally,

$$
\begin{aligned}
\int_{0}^{\infty}\left(H(\lambda) \lambda^{-1}\right)^{2} d \lambda & =\sum_{N=1}^{\infty}\left(\sum_{n=N}^{\infty} a_{n} n^{-1 / 2}\right)^{2} \\
& =\sum_{n=1}^{\infty} a_{n}^{2}+2 \sum_{(m, n)=1, n<m}(n / m)^{1 / 2} \sum_{k=1}^{\infty} a_{n k} a_{m k} \\
& =\sum_{n=1}^{\infty} a_{n}^{2}=1 .
\end{aligned}
$$

Obviously $|\phi(i t)| \leqq 1$ a.e. Hence

$$
\int_{0}^{\infty}\left(H(\lambda) \lambda^{-1}\right)^{2} d \lambda=\frac{1}{2 \pi} \int_{-\infty}^{\infty} \frac{|\phi(i t)|^{2}}{1 / 4+t^{2}} d t \leqq \frac{1}{2 \pi} \int_{-\infty}^{\infty} \frac{1}{1 / 4+t^{2}} d t=1
$$

Accordingly, if we can justify the formal rearrangements in equation 7.12, or give criteria for this purpose, it would follow from equation 7.13 that $|\phi(i t)|=1$ a.e. In this connection another relation may be noted, namely $\sum \Delta\left((H(\lambda))^{2} \lambda^{-z}\right)=\sum a_{n}^{2}$ where we sum over the jumps of $H(\lambda) \lambda^{-1}$.

We are interested in the relation between property $\mathrm{W}$ and the class $K$. The following theorem sums up some consequences of $W$.

TheOREM 7.4. If (a) $H(\lambda) \lambda^{-1}$ satisfies relation $\mathrm{W}$, then (A) $\sum a_{n} n^{-8}$ is convergent for $R(z)>0$, (B) $|\phi(s+i t)|=O\left(|t|^{1 / 2}\right), t \rightarrow \infty, 0<s<1 / 2$, (C) $\phi(s+i t) / 2^{-1}$ $-s-i$ converges to $\phi(i t) / 2^{-1}-i t,|\phi(i t)|=1$, a.e. in the norm topology of $L_{2}(-\infty$, $\infty)$, (D) $M\{\phi(s+i t)\}$ exists for $s>0$, and (E) $|\phi(s+i t)|^{2} /\left((1 / 2-s)^{2}+t^{2}\right)^{a}$ belongs to $L_{1}(-\infty, \infty)$ for $\alpha>1 / 2$ and $0<s<1 / 2$.

Evidently

$$
\int_{0}^{\infty}\left(H(\lambda) \lambda^{s-1}\right)^{2} d \lambda \leqq \int_{0}^{1}\left(H\left(\mu^{-1}\right) \mu^{-s}\right)^{2} d \mu+\int_{1}^{\infty}\left(H\left(\mu^{-1}\right) \mu^{-s}\right)^{2} d \mu .
$$

Since $H\left(\mu^{-1}\right)$ is constant on intervals of unit length, we infer it is uniformly 
bounded in absolute value. Hence the first integral on the right-hand side of equation 7.14 is finite for $0 \leqq s<1 / 2$. The second integral is a decreasing function of $s$ and hence is finite for $s \geqq 0$. Accordingly $H(\lambda) \lambda^{-1} \in L_{2}(0, \infty)$ for $0 \leqq s<1 / 2$. It is easy to show that

$$
\int_{0}^{\infty}\left|H(\lambda) \lambda^{r-1} \lambda^{i t-1 / 2}\right| d \lambda
$$

exists for $0<s<1 / 2$. A straightforward argument using $H_{N}\left(\mu^{-1}\right)$ then shows that the $\mathrm{M}$ transform of $H(\lambda) \lambda^{s-1}$ is $\phi(s+i t) / 2^{-1}-s-i t$ in this range. This is essentially conclusion (A) of the theorem. Moreover, $\left|\phi(s+i t) / 2^{-1}-s-i t\right|$ is dominated by the integral in equation 7.15. This implies $|\phi(s+i t)|=O(|t|)$ $t \rightarrow \infty$. The method used in demonstrating Theorem 7.1 shows $\phi(s+i t) / 2^{-1}-s$ $-i t$ converges in the norm topology of $L_{2}(-\infty, \infty)$ to $\phi(i t) / 2^{-1}-i t$. The central result of the Watson theory of general transforms guarantees that $|\phi(i t)|=1$ as a consequence of condition (a). Thus conclusion (C) is justified.

In view of equation 7.15, $\phi(s+i t) / 2^{-1}-s-i t$ is analytic in $0<s<1 / 2$ $[13$, p. 247 $]$ and accordingly $\phi(s+i t)$ is analytic for $s>0$. Moreover, for $s$ sufficiently large, $\sum a_{n} n^{-(\bullet+i t)}$ is uniformly convergent in $t$ and hence is a.p. Together with the italicized order relations on $|\phi(s+i t)|$, these facts ensure that $M\{\phi(z)\}$ exists for $s>0[2$, p. 164]. This is conclusion (D) and implies, since we are dealing with ordinary Dirichlet series, that actually $|\phi(s+i t)|=O\left(|t|^{1 / 2}\right), t \rightarrow \infty, s>0[7, \mathrm{p}$. 307]. It is then plain that $|\phi(z)|^{2} /|1 / 2-z|^{2 \alpha} \in L_{1}(-\infty, \infty), 0<s<1 / 2$, for $\alpha>1$. However, to show $\alpha>1 / 2$ is sufficient requires more precise analysis. Thus

$$
\begin{aligned}
\int_{-T}^{T} \frac{|\phi(s+i t)|^{2}}{\left((1 / 2-s)^{2}+t^{2}\right)^{\alpha}} d t & \\
= & 2 \int_{0}^{T}|\phi(s+i t)|^{2} \int_{t}^{\infty}\left|\frac{d}{d x}\left((1 / 2-s)^{2}+x^{2}\right)^{-\alpha}\right| d x d t \\
= & 2 \int_{0}^{T} x\left|\frac{d}{d x}\left((1 / 2-s)^{2}+x^{2}\right)^{-\alpha}\right| \frac{1}{x} \int_{0}^{x}|\phi(s+i t)|^{2} d t d x \\
& +2 T \int_{T}^{\infty}\left|\frac{d}{d x}\left((1 / 2-s)^{2}+x^{2}\right)^{-\alpha}\right| \frac{1}{T} \int_{0}^{T}|\phi(s+i t)|^{2} d t d x .
\end{aligned}
$$

We have shown that $|\phi(s+i t)|$ is bounded in any finite $t$ interval and $M\{\phi(s+i t)\}, s>0$, exists. Hence $(1 / x) \int_{0}^{x}|\phi(s+i t)|^{2} d t<L<\infty$ for all $x$. Accordingly the right-hand side of equation 7.16 is inferior to

$$
4 L T /\left((1 / 2-s)^{2}+T^{2}\right)^{\alpha}+2 L \int_{0}^{T}\left((1 / 2-s)^{2}+x^{2}\right)^{-\alpha} d x .
$$

Since $\alpha>1 / 2$ the first term goes to 0 with $T \rightarrow \infty$. The second remains finite. 
Hence the limit as $T \rightarrow \infty$ of the left-hand side of equation 7.16 exists and the assertion in (E) is established. In a similar way we can show

$$
L_{s \rightarrow 0+} L_{T \rightarrow \infty} \sup _{0 \leqq t \leqq T} \frac{1}{2 t} \int_{-t}^{t}|\phi(s+i t)|^{2} d t \geqq 1
$$

where $\infty$ is now included as a possible value for the left-hand side.

Remark. In view of the next theorem one should expect that the $\mathrm{W}$ property for $H(\lambda) \lambda^{-1}$ might imply some (if not all) of the hypotheses of Theorem 6.1 , and certainly more than we have obtained above.

Theorem 7.5. If $H(\lambda) \lambda^{-1}$ satisfies $\mathrm{W}$ and (a) $\left\{a_{n}\right\} \in U$ or (b) $\left\{a_{n}\right\}$ has a one-element base, then $\left\{a_{n}\right\} \in K$.

It is not difficult to show that under the present hypotheses the function $\phi(i t)$, occurring in (C) of the previous theorem, is here $\sum a_{n} n^{-i t}$. In the case of (b) we require Theorem 10.3 to establish this. Hence Theorem 3.1 yields the assertion of the theorem.

8. Examples. An example was given in [1] of a solution with isolated (essential) singularities on the axis of imaginaries. The question of the existence of a solution with $R(z)=0$ as a natural boundary was left open. For a one-element base (and hence for a finite base) the existence of such a solution can be asserted on the basis of the representation in equation 10.07 , cf. $\$ 10$. It seems of interest, however, to treat a case with an infinite base directly.

Let $C^{m}=\left\{C_{n}^{m} \mid n=1,2 \cdots\right\} \in l_{2}$. It is convenient to define a partial norm by $\left(\sum_{n=1}^{N} C_{n}^{2}\right)^{1 / 2}=\|C\|_{N}$. We proceed with the definition of $\phi(z)$. Let

$$
\phi_{m}(z)=\left(A_{m}+\overrightarrow{p_{m}^{2}}\right) /\left(1+A_{m} \overrightarrow{p_{m}^{-z}}\right), \quad \phi^{m}(z)=\prod_{1}^{m} \phi_{j}(z) .
$$

The coefficient sequence $\left\{a_{n}^{m}\right\}$ in the Dirichlet expansion of $\phi^{m}(z)$ is denoted by $a^{m}$. We require $p_{m+1}>N(m)$, where $N(m)$ is subject to the restrictions

$$
\left\|a^{m}\right\|_{N(m)} \geqq\left(2^{m}-1\right) / 2^{m}, \quad N(m)>N(m-1) .
$$

The $p_{m}$ 's need not be primes. We choose $\left\{A_{m}\right\}$ to satisfy $0<1-A_{m+1}$ $<(N(m))^{-2}$. Plainly

$$
a_{n}^{m}=A_{m}\left(a_{n}^{m-1}\right), \quad 1 \leqq n \leqq N(m-1) .
$$

Hence

$$
\left\|a^{m+1}-a^{m}\right\|_{N(m)} \leqq 1-A_{m+1} .
$$

It is not difficult to show that

$$
\left\|a^{m+r}-a^{m}\right\|_{N(m)} \leqq 2 / m .
$$


In view of equation 8.02 , for fixed $N$,

$$
L_{m, m^{\prime} \rightarrow \infty}\left\|a^{m}-a^{m^{\prime}}\right\|_{N}=0 .
$$

Now $\left\|a^{m}\right\|=1$ and it is easy to see that equation 8.02 implies

$$
L_{m \rightarrow \infty} a_{n}^{m}=a_{n}, \quad n=1,2, \cdots \text {. }
$$

This is to say, $a^{m}$ converges weakly $\left[8\right.$, p. 137] to $a \in l_{2}$. Plainly,

$$
\left\|a-a^{m}\right\|_{N(m)} \leqq 2 / m \text {. }
$$

Moreover, in view of equations 8.01 and 8.02 ,

$$
\begin{aligned}
\|a\| & \geqq\|a\|_{N(m)} \\
& \geqq\|a\|_{N(m)}-\left\|a-a^{m}\right\|_{N(m)} \\
& \geqq\left(2^{m}-1\right) / 2^{m}-2 / m .
\end{aligned}
$$

Therefore $\|a\| \geqq 1$. We may interpret $a$ and $a^{m}$ as elements of the space of linear functionals (again $l_{2}$ ) over $l_{2}$. We have then $[8$, p. 123]

$$
\|a\| \leqq \liminf _{m \rightarrow \infty}\left\|a^{m}\right\|=1 \text {. }
$$

Hence $\|a\|=1$ and therefore $a$ is a norm limit as well $[8$, p. 140]. Moreover $a \in K$ for the set of solutions in $K$ is closed in $l_{2}$ [1, Theorem 11.1].

We assert that for $\eta>0$ and some choice of $N$

$$
P_{N}(z)=\prod_{N}^{\infty} \phi_{j}(z)
$$

converges absolutely and uniformly in $R(z) \geqq \eta$. Write

$$
\phi_{j}(z)=1+\left(A_{j}-1\right)\left(\left(1-\overrightarrow{p_{j}^{-z}}\right) /\left(1+A_{i} p_{j}^{-z}\right)\right) .
$$

Choose $M$ such that

$$
-\log \left|A_{i}\right| / \log p_{i}<\eta
$$$$
j>M \geqq N \text {. }
$$

This can obviously be done. For fixed $z, R(z) \geqq \eta$,

$$
\left|\left(1-\overrightarrow{p_{i}}\right) /\left(1+A_{i} \overrightarrow{p_{j}}\right)\right|<1, \quad j=1,2, \cdots \text {. }
$$

Since $\left|A_{j}-1\right|<j^{-2}$ it follows that

$$
\left|\phi_{j}(z)\right| \leqq 1+j^{-2} \text {. }
$$

The convergence of $\sum_{j=M}^{\infty} j^{-2}$ then guarantees the absolute and uniform convergence of $P_{M}(z), R(z) \geqq \eta>0$. It is clear that

$$
\phi(z)=\sum a_{n} n^{-z}=\phi^{M-1}(z) P_{M}(z), \quad R(z) \geqq \eta,
$$

and hence $\phi(z)$ has zeros at 


$$
-\log \left|A_{j}\right| / \log p_{i} \pm 2 k \pi i / \log p_{j} .
$$

As $j \rightarrow \infty$ the lines, $R(z)=-\log \left|A_{j}\right| / \log p_{j}$, containing zeros approach $R(z)=0$ and the vertical spacing of the zeros goes to 0 . Hence every point on $R(z)=0$ is the limit point of values of $z$ for which $\phi(z)=0$. Hence $\phi(z)$ is not analytic at any point on $R(z)=0$. We state our results in the form of a theorem.

THEOREM 8.1. The $\phi(z)$ defined above is a solution with $R(z)=0$ as a natural boundary.

Remark. An interesting property of the solution constructed above is that $a_{1} \neq 0$ for $\eta=p_{j}$. (Actually much more is true as will appear presently.) Thus from equation 7.01 it follows that $a_{i}^{m} \neq 0$ for some $i \leqq N(m)$. For any $l>m$, since $p_{l}>N(m)$,

$$
\begin{aligned}
\left|a_{i}^{l}\right| & =\left|a_{i}^{m}\right| \prod_{m+1}^{l} A_{i} \\
& \geqq\left|a_{i}^{m}\right|\left|A_{1}\right| \prod_{2}^{\infty}\left(1-(N(j))^{-2}\right) \\
& \geqq\left|a_{i}^{m}\right|\left|A_{1}\right| / 2 .
\end{aligned}
$$

Since $a_{i}^{m}$ converges to $a_{i}$ it is plain that $a_{i} \neq 0$. The form of $\phi^{j}(z)$ indicates that $a_{n}^{j} \neq 0, n=p_{j}$. Thus $a_{n} \neq 0, n=p_{j}$.

The second example uses products of the form of the solutions given in $[1, \S 13]$. Its interest lies in part in the fact that here there are no zeros in $R(z)>0$.

Let $A_{m}$ be positive and inferior to $m^{-6}$. Let $\left\{n_{j}\right\}$ be a monotone increasing sequence of positive integers. We assert that

$$
\phi(z)=\exp -\sum_{l=1}^{\infty} A_{l} \operatorname{coth}\left(z\left(\log n_{i}\right) / 2\right)
$$

belongs to $K$ and has $R(z)=0$ as a natural boundary.

Since limits of uniformly convergent series of a.p. functions are a.p., it is not difficult to see that $\phi(z)$ is a.p. for $R(z)>0$.

We have

$$
|\phi(s+i t)|^{2} \sim \exp -2 \sum_{l=1}^{\infty} A_{l} h_{l}(s, t)
$$

where

$$
h_{l}(s, t)=\left(1-n_{l}^{-2 g}\right) /\left(\left(1-\overrightarrow{n_{l}}\right)^{2}+2 n_{l}^{-s} \sin ^{2}\left(t\left(\log n_{l}\right) / 2\right)\right) .
$$

We observe that

$$
\sum_{l=m+1}^{\infty} A_{l} h_{l}(s, t) \leqq 2\left(1-m^{-s}\right)^{-1} \sum_{l=m+1}^{\infty} A_{l} \leqq\left(1-m^{-s}\right)^{-1} m^{-s} .
$$


Let $\epsilon=m^{-1}$. The right-hand side of equation 8.05 is dominated by $\epsilon$ provided $s$ is restricted to satisfy

$$
n_{m}^{*}-1 \geqq m^{-4} \text {. }
$$

This gives a lower bound for $s$. An upper bound is obtained from the following condition, which plays a role in our subsequent considerations:

$$
n_{m}^{8}-1 \leqq\left(\pi^{2} m^{3}\right)^{-1} \text {. }
$$

Plainly, equations 8.06 and 8.07 are consistent for large values of $m$.

Consider the subset of $0 \leqq t \leqq \pi / 2$ such that

$$
h_{m}(s, t)<\epsilon .
$$

This condition is surely satisfied if

Obviously

$$
\sin ^{2}\left(t\left(\log n_{m}\right) / 2\right) \geqq\left(n_{m}^{*}-1\right) \epsilon^{-1} .
$$

$$
\sin \theta \geqq 2 \theta / \pi,
$$$$
0 \leqq \theta \leqq \pi / 2 .
$$

Then, in view of equation 8.07 and the choice $\epsilon=m^{-1}$, it is easy to see that equation 8.08 is valid when $t$ is restricted to satisfy

$$
\pi / \log n_{m}>t \geqq\left(m \log n_{m}\right)^{-1} \text {. }
$$

We remove intervals of length $2\left(m \log n_{m}\right)^{-1}$ centered at the points

$$
t=2 k \pi / \log n_{m}, \quad k=0, \pm 1, \cdots .
$$

Then, clearly, equation 8.08 holds for all $t$ values foreign to the intervals removed. Consider the set $S(T):-T \pi \leqq t \leqq T \pi$. There are at most [T $\left.\log n_{m}\right]$ values of $t$ satisfying equation 8.09 . Hence the measure of the set removed is at most

$$
2 T\left[\log n_{m}\right] / n_{m} \log n_{m} .
$$

Evidently

$$
\frac{\dot{n_{m}}-1}{\log n_{m}} \geqq \frac{\dot{n_{j}}-1}{\log n_{i}},
$$

We conclude that equation 8.08 holds with $j$ replacing $m, j<m$, if

$$
\pi / \log n_{j}>t \geqq \pi\left(\left(n_{m}^{*}-1\right) m\right)^{1 / 2} /\left(\log n_{m} \log n_{j}\right)^{1 / 2}
$$

or, in view of equation 8.07 ,

$$
\pi / \log n_{j}>t \geqq\left(m\left(\log n_{m} \log n_{j}\right)^{1 / 2}\right)^{-1} .
$$

An argument, similar to that developed in the consideration of $h_{m}(s, t)$, shows 
that $h_{j}(s, t)<\epsilon, j<m$, if we remove from $S(T)$ a subset of measure at most

$$
\left[2 T \log n_{m}\right] / m\left(\log n_{m} \log n_{j}\right)^{1 / 2} \text {. }
$$

Hence the subset $S^{\prime}(T), S^{\prime}(T) \subset S(T)$, for which $h_{j}(s, t)<\epsilon, j=1, \cdots, m$, has measure at least

$$
2 \pi T\left(1-\left(m \log n_{m}\right)^{-1} \sum_{j=1}^{m}\left(\log n_{j}\right)^{1 / 2}\right) \geqq 2 \pi T\left(1-\left(\log n_{m}\right)^{-1}\right) .
$$

For $t \in S^{\prime}(T)$ we have

$$
\sum_{l=1}^{m} A_{l} h_{l}(s, t) \leqq \sum_{l=1}^{m}\left(m l^{6}\right)^{-1} \leqq 2 / m .
$$

Accordingly for $t \in S^{\prime}(T)$, and $s$ satisfying equations 8.06 and 8.07 , we have

$$
\begin{gathered}
|\phi(s+i t)|^{2} \geqq e^{-\delta / m}, \\
M\{\phi(s+i t)\} \geqq e^{-\delta / m}\left(1-\left(\pi \log n_{m}\right)^{-1}\right) .
\end{gathered}
$$

As $m \rightarrow \infty$, the right-hand side of equation 8.11 goes to 1 . It is well known that $M\{\phi(s+i t)\}$ is monotonic nondecreasing as $s \rightarrow 0+$. Since $1 / m=\epsilon \rightarrow 0 \mathrm{im}$ plies $s \rightarrow 0+$ we have shown that

$$
L_{s \rightarrow 0+} M\{\phi(s+i t)\} \geqq 1 .
$$

On the other hand, since $h_{j}(s, t)$ is non-negative, we have $|\phi(s+i t)| \leqq 1, s>0$, and therefore $M\{\phi(s+i t)\}<1$. Hence

$$
L_{s \rightarrow 0+} M\{\phi(s+i t)\}=1 .
$$

It follows from Theorem 6.1 that $\phi(s+i t)$ is a solution.

Suppose $R(z)=0$ is not a natural boundary. Then for some open interval, $V$, on $R(z)=0, \phi(z)$ is analytic. Accordingly

$$
\phi(i t)=L_{s \rightarrow 0+} \phi(s+i t),
$$

The set $Q=\left\{t \mid t=2 k \pi / \log n_{l}, k=0, \pm 1, \cdots, l=1,2, \cdots\right\}$ is a denumerable dense set on $R(z)=0$. For $t \in V \cap Q$ it is easy to see that

$$
L_{s \rightarrow 0+}|\phi(s+i t)|=0 \text {. }
$$

Hence, by continuity, $\phi(i t)=0$ for $t \in V$. Then Theorem 4.6 guarantees $\phi(s+i t)=0, s>0$. This is plainly absurd.

It is of interest to show a little more, namely that for each $\rho>0$ there is a set $\Gamma_{\rho}$ such that $\phi(s+i t)$ converges uniformly as $s \rightarrow 0+, t \notin \Gamma_{\rho}$, to a function $\phi(i t)$ of modulus 1 and, with $\mu$ denoting the measure function,

$$
\mu\left\{\Gamma_{\rho} \cap\{t \mid-T \leqq t \leqq T\} / 2 T=O(\rho), \quad T \rightarrow \infty .\right.
$$

Since $s$ is not restricted to a sequence of values, obviously something more 
than Egoroff's theorem is involved. In order not to complicate the developments, we prove our assertion under the restriction $n_{l}<l^{k}$ for some positive fixed $k$.

Since $\phi(s)$ is real we shall write $(\phi(z) / \phi(\bar{z}))^{1 / 2}$ for $\exp i \arg \phi(z)$. It is easy to verify that with $z=s+i t, z^{\prime}=s^{\prime}+i t$,

$$
\left|\left(\frac{\phi(z)}{\phi(\bar{z})}\right)^{1 / 2}-\left(\frac{\phi\left(z^{\prime}\right)}{\phi\left(\bar{z}^{\prime}\right)}\right)^{1 / 2}\right|=\left|1-\exp -i \theta\left(s, s^{\prime} ; t\right)\right|,
$$

where

$$
\theta\left(s, s^{\prime} ; t\right)=\sum_{l=1}^{\infty} 2 A_{l}\left(n_{l}^{-s^{\prime}}-\overrightarrow{n_{l}^{-s}}\right)\left(n_{l}^{-s^{\prime}}-1\right) \sin \left(t \log n_{l}\right) / D_{l}(s, t) D_{l}\left(s^{\prime}, t\right)
$$

and

$$
D_{l}(s, t)=\left(1-\overrightarrow{n_{l}}\right)^{2}+2 \overrightarrow{n_{l}} \sin ^{2}\left(t\left(\log n_{l}\right) / 2\right) .
$$

Then

$$
\begin{aligned}
& \left|\theta\left(s, s^{\prime} ; t\right)\right| \\
& \quad \leqq \sum_{l=1}^{\infty}\left|A_{l}\left(\overrightarrow{n_{l}}-\overrightarrow{n_{l}}\right)\left(1-n_{l}^{-(\imath+\imath)}\right) / 2 n_{l}^{-\left(\imath+\theta^{\prime}\right)} \sin ^{4}\left(t\left(\log n_{l}\right) / 2\right)\right| .
\end{aligned}
$$

Choose positive numbers $\delta$ and $\gamma$ subject to $4 \delta+\gamma<1$. Enclose each of the points $2 \pi k / \log n_{l}, k=0, \pm 1, \pm 2, \cdots, l=1,2, \cdots$, by an interval centered at this point of length $2 \rho / l^{1+\delta} \log n_{l}$. Denote the union of these intervals by $\Gamma_{\rho}$. Hence the measure of $\Gamma_{\rho} \cap\{-T \pi \leqq t \leqq T \pi\}$ is less than $\rho B T$ where $B$ is independent of $\rho$ and $T$.

We have

$$
\left|\sin ^{4}\left(t\left(\log n_{l}\right) / 2\right)\right| \geqq\left(\rho / \pi l^{1+8}\right)^{4},
$$

$t \notin \Gamma_{\rho}$.

In view of our restrictions on $\delta, \gamma, A_{l}$, and $n_{l}$ we can choose $s_{\rho}$ so that for $0<s^{\prime} \leqq s<s_{\rho}$ we have

$$
A_{l}\left(n_{l}^{-b^{\prime}}-\overrightarrow{n_{l}}\right)\left(n_{l}^{s+\iota^{\prime}}-1\right) / 2 \sin ^{4}\left(t\left(\log n_{l}\right) / 2\right)<\rho l^{-1-\gamma}, \quad l=1,2, \cdots .
$$

Hence

$$
\left|\theta\left(s, s^{\prime} ; t\right)\right| \leqq c_{\rho} .
$$

Accordingly the left-hand side of equation 8.12 goes to 0 uniformly in $t, t \notin \Gamma_{\text {p }}$ for every $\rho>0$. Now it can easily be shown that

$$
\left|\phi(z)-\phi\left(z^{\prime}\right)\right| \leqq\left|\phi(z)-\phi\left(z^{\prime}\right)\right|+\left|\phi\left(z^{\prime}\right)\right|\left|\left(\frac{\phi(z)}{\phi(\bar{z})}\right)^{1 / 2}-\left(\frac{\phi\left(z^{\prime}\right)}{\phi\left(\bar{z}^{\prime}\right)}\right)^{1 / 2}\right| .
$$

The argument for the first part of the discussion of this example and equation 8.15 show that the left-hand side of equation 8.15 goes to 0 uniformly 
in $t, t \notin \Gamma_{\rho}$ as $s \rightarrow 0+$. That is to say, $L_{s \rightarrow 0+} \phi(s+i t)=\phi(i t)$ uniformly in $t$ for $t \notin \Gamma_{\rho}$ and $|\phi(i t)|=1$.

Remark. A gap theorem observation shows that if $\phi(z) \in K$ and has a finite base then $a_{n} a_{m} \neq 0$, for some pair $n \neq m$, implies that $|w|=1$ is a natural boundary for $\sum a_{n} w^{n}$. It seems likely that the second example above or some modification would show that $\phi(z) \in K$ is not sufficient. It is of interest to remark that in his Theorem 14.1 of [1] the writer proved that for a class of Dirichlet series including $K$ the hypothesis $a_{n} a_{m} \neq 0$ ensures a singularity at $w=1$ for $\sum a_{n} w^{n}$. The most interesting question, however, is whether all solutions on a nonfinite base have $R(z)=0$ as the natural boundary.

9. Completeness of sets of functions. The systems in this section are of the form $\{f(n x)\}$. All orthogonality relations are now waived.

As a preliminary to the main results of this section we remark that a wide variety of completeness theorems may be obtained if one imposes analyticity conditions on $f(x+i y)$, following the Szász idea [14] of associating the completeness problem with that of the zeros of certain integrals. We give a single theorem to illustrate this aspect. Our result pivots on the use of Carlson's theorem, but obviously wide generalization is possible according to the analogues of Carlson's theorem employed. It will be observed that periodicity of $f(x)$ is no longer assumed.

TheOREM 9.1. Suppose (a) $f(0)=0$, (b) $f(w), w=x+i y$, is a regular analytic function for $R(w) \geqq 0$, (c) $f^{l}(0) \neq 0$ for all positive integers $l$, and (d) $|f(w)|=O\left(e^{k|w|}\right), k<1 / 2$, for $R(w)>0$. Then $\{f(n x)\}$ is complete in $L_{2}(0,2 \pi)$.

Let $h(x) \in L_{2}(0,2 \pi)$. Then

$$
F(w)=\frac{1}{\pi} \int_{0}^{2 \pi} h(v) f(w v) d v
$$

vanishes for $w=0,1,2, \ldots$. Manifestly

That is to say

$$
|F(w)| \leqq A\left(\int_{0}^{2 \pi}|h(v)|^{2} d v\right)^{1 / 2} e^{2 \pi k|w|}, \quad R(w)>0 .
$$

$$
|F(w)|=O\left(e^{h|w|}\right), \quad|y| \rightarrow \infty, \quad h=2 \pi k<1 / 2 .
$$

Hence Carlson's theorem implies

$$
F(w) \equiv 0, \quad 0=F^{l}(0)=\int_{0}^{2 \pi} h(v) v^{l} f^{l}(0) d v .
$$

In view of (c) this implies that $h(x)$ is orthogonal to all Legendre polynomials and hence $h(x)=0$ a.e. Accordingly $\{f(n x)\}$ is closed and thus is complete in $L_{2}(0,2 \pi)$.

We find the following conventions convenient: If $\left\{b_{n} \mid n=2, \cdots\right\}$ is a 
sequence belonging to $l_{2}$ then

$$
B_{i, j}=B_{I, J}=\sum_{k=1}^{\infty} b_{I k} b_{J k}
$$

where $I=i /(i, j)$ and $J=j /(i, j)$. The sequence $\left\{c_{n}^{M} \mid n=1, \cdots, M\right\}$ is a minimizing sequence associated with $F(x)$ and $\left\{g_{n}(x)\right\}$ if

$$
\left\|F(x)-\sum_{n=1}^{M} c_{n}^{M} g_{n}(x)\right\| \leqq\left\|F(x)-\sum_{n=1}^{M} c_{n} g_{n}(x)\right\|
$$

for all choices of $\left\{c_{n} \mid n=1, \cdots, M\right\}$ where now the norm is the general $L_{2}$ norm.

We now establish two simple results of general interest. Let

$$
f(x) \in L_{2}(0,-\pi, \pi)
$$

with

$$
f(x) \sim \sin x+\sum_{n=2}^{\infty} b_{n} \sin n x .
$$

We say a sequence is minimal if no one of its members is in the closed linear extension of the others.

TheOREM 9.2. The sequence $\{f(n x)\}$ is minimal.

TheOREM 9.3. For a minimizing sequence we have

$$
\left\|\sin x-\sum_{1}^{M} c_{n}^{M} f(n x)\right\|^{2}=\left|1-c_{1}^{M}\right| \text {. }
$$

Suppose the first theorem untrue. Then for some $N$ we must have $f(N x)$ in the closed linear manifold spanning $\{f(n x) \mid n \neq N\}$. Consider

$$
f(N x)-\sum^{\prime} c_{i} f(i x) \sim \sum_{j=1}^{\infty} A_{j} \sin j x
$$

where the prime indicates the term $i=N$ is omitted. If the squared norm of the left-hand side can be made arbitrarily small, this must certainly be true for $D_{N}$ where

$$
D_{N}=\sum_{j=1}^{N} A_{j}^{2}
$$

$$
A_{i}=\sum_{l \mid j} c_{l} b_{j / l}, b_{1}=1 \text {. }
$$

Evidently, then, at least one $c_{j}$ differs from 0 for $1 \leqq j \leqq N-1$. Clearly $D_{N}$ is non-negative and takes on its minimum for some finite $c_{j}$ values which we denote by $\left\{c_{j}^{\prime} \mid j=1, \cdots, N-1\right\}$. Let $k$ be the first index for which $c_{j}^{\prime}$ is non- 
zero. Clearly it follows that $D_{N}$ is always at least $c_{\boldsymbol{k}}^{\prime 2}$. This establishes the assertion of the theorem.

For the second theorem we need only observe that the sequence $\left\{c_{n}^{M}\right\}$ is defined by

$$
\left[\sin x-\sum_{n=1}^{M} c_{n}^{M} f(n x), f(j x)\right]=0, \quad j=1, \cdots, M
$$

Since

$$
[\sin x, f(j x)]=0, \quad i \neq 1,
$$

it follows that

$$
\left\|\sin x-\sum_{1}^{M} c_{n}^{M} f(n x)\right\|^{2}=\left[\sin x-\sum_{n=1}^{M} c_{n}^{M} f(n x), \sin x\right]=\left|1-c_{1}^{M}\right| .
$$

The result is of interest in that it suggests a criterion for completeness of $\{f(n x)\}$.

THEOREM 9.4. If $f(x) \sim \sin x-\sum_{n=2}^{\infty} b_{n}$ sin $n x$ where $\left\{b_{n}\right\} \in l_{2}$ and if $\sum_{(i, j)-1}\left|B_{i, j}\right|<1$ then $(\mathrm{A})\{f(n x)\}$ is complete in $L_{2}(0 ;-\pi, \pi)$. that

If $\left\{c_{n} \mid n=1, \cdots\right\} \in l_{2}$ then it may be shown in a straightforward manner

$$
\left\|\sum_{k=1}^{N} c_{k}(f(k x)-\sin k x)\right\|^{2} \leqq \sum_{i, j=1}^{N}\left|c_{i} c_{j} B_{i, j}\right| .
$$

We prepare for application of the Schwarz inequality by writing the righthand side of equation 9.01 in the form

$$
\left.\sum_{i=1}^{N} \sum_{j=1}^{N}\left|c_{i}\right| B_{i j}\right|^{1 / 2} c_{j}\left|B_{i j}\right|^{1 / 2} \mid
$$

Hence we have the bounds

$$
\sum_{i=1}^{N} \sum_{j=1}^{N}\left|c_{j}\right|^{2}\left|B_{i, j}\right| \leqq \sum_{n=1}^{N}\left(c_{n}\right)^{2} \sup _{1 \leqq i<\infty} \sum_{i=1}^{\infty}\left|B_{i, j}\right| \leqq \infty .
$$

We show now that, for arbitrary $N$,

$$
\sup _{1 \leqq i<\infty} \sum_{i=1}^{\infty}\left|B_{i, j}\right| \geqq \sum_{(i, j)=1}^{N}\left|B_{i, j}\right|
$$

Indeed let $M$ be the L.C.M. of the integers $1,2, \cdots, N$. Then

$$
B_{M s / r, M}=B_{M r / s, M}=B_{r, s}, \quad(r, s)=1 ; r, s=1, \cdots, N .
$$

Hence, for $j=M$, all terms in the sum on the right-hand side of equation 9.02 are included in the sum on the left-hand side. On the other hand, we can show that 


$$
\sum_{i=1}^{\infty}\left|B_{i, j}\right| \leqq \sum_{(i, j)=1}^{\infty}\left|B_{i, j}\right| .
$$

This follows from the observation that a term $B_{i, j}=B_{I, J}, I J>1$, can enter at most two times in the left-hand sum. This term is counted exactly twice in the right-hand sum. Obviously the term $B_{1,1}$ is counted once in each sum. In consequence of equations 9.02 and 9.03 we have

$$
\sup _{1 \leqq j<\infty} \sum_{i=1}^{\infty}\left|B_{i, j}\right|=\sum_{(i, j)=1}^{\infty}\left|B_{i, j}\right| .
$$

That is to say,

$$
L_{N \rightarrow \infty}\left\|\sum_{n=1}^{N} c_{n}(f(n x)-\sin n x)\right\|^{2} \leqq \theta^{2} \sum_{1}^{\infty} c_{n}^{2}, \quad 0 \leqq \theta<1 .
$$

Since $\{\sin n x\}$ is complete, it follows from a well known theorem due to Paley and Wiener $\left[15\right.$, p. 100] that $\{f(n x)\}$ is complete in $L_{2}(0 ;-\pi, \pi)$.

CoRollary. If $f(x) \sim \sin x-\sum_{n=2}^{\infty} b_{n} \sin n x$ with $\sum_{n=2}^{\infty}\left|b_{n}\right|<1$ then $\{f(n x)\}$ is complete in $L_{2}(0 ;-\pi, \pi)$.

This is a trivial consequence of the inequality $\sum_{(i, j)-1}\left|B_{i, j}\right| \leqq\left(\sum_{n=2}^{\infty}\left|b_{n}\right|\right)^{2 \cdot}$

TheOREM 9.5. If $f(x) \sim \sin x-\sum_{n=2}^{\infty} b_{n} \sin n x$ and $T$ is the Toeplitz matrix for $\left\{b_{n} \mid b_{1}=0\right\}$, then, if $T$ is bounded by $\theta, 0<\theta<1$, the sequence $\{f(n x)\}$ is complete in $L_{2}(0 ;-\pi, \pi)$.

We observe that $T T^{*}$ is the matrix $\left(\left(B_{i, j}\right)\right)$. Moreover, since $T$ has the bound $\theta$ so has $T^{*}[5, \mathrm{p} .132]$. Accordingly $\left(\left(B_{i, j}\right)\right)$ has the bound $\theta^{2}$. This implies $[5$, p. 123] the hypothesis of the Paley-Wiener theorem.

The following comments give proper perspective. The proofs are merely indicated. If $\{f(n x)\}$ is complete, then $\{f(n x)\}$ is closed. Therefore $L_{M \rightarrow \infty}\left\|\sin x-\sum_{n=1}^{M} c_{n}^{M} f(n x)\right\|=0$. Let

$$
c^{M}=\left(c_{1}^{M}, \ldots, c_{M}^{M}, 0,0, \cdots\right) .
$$

Since $\{f(n x)\}$ is minimal, it follows that

$$
L_{M \rightarrow \infty}^{M} C_{n}^{M}=d_{n}
$$$$
n=1,2, \cdots
$$

Moreover

$$
\psi_{M}(x)=\sin x-\sum_{n=1}^{M} d_{n} f(n x)
$$

is in the closed linear extension of $\{f(j x) \mid j=M+1, \cdots\}$ (this is plainly equivalent to orthogonality of $\psi_{M}(x)$ to $\left.\sin j x, j=1, \cdots, M\right)$ and $\left\{d_{n}\right\}$ is uniquely determined. The idea of the proof occurs in rudimentary form in 
[16, Lemma 1] but for complete covering theorems we need [17]. The sequence $\left\{d_{n}\right\}$ defined above will be referred to as a weak sequence. The PaleyWiener theorem implies in our case that for suitable $\left\{e_{n}\right\} \in l_{2}$

P.W.:

$$
L_{N \rightarrow \infty}\left\|\sin x-\sum_{n=1}^{N} e_{n} f(n x)\right\|=0 .
$$

We can show that actually $e_{n}=d_{n}$. The proof hinges on the observation that the defining equation [15, Equation 29.23] determines $e_{n}$ in terms of $\left\{b_{j} \mid j=2, \cdots, n\right\}$ so that the orthogonality relations above are satisfied.

The remaining theorems of this section are more in keeping with the spirit of the developments of this paper.

TheOREM 9.6. If $f(x) \sim \sin x-\sum_{n-2}^{\infty} b_{n} \sin n x,\left\{b_{n}\right\} \in U$, and for some $\theta$, $0<\theta<1, L_{N \rightarrow \infty} \sum_{(i, j)=1} B_{i, j} \cos (t(\log i-\log j)) \leqq \theta^{2}$, then $\{f(n x)\}$ is complete.

This theorem is connected with Theorem 9.5 since the boundedness of the Toeplitz matrix implies boundedness of the associated Dirichlet series (with the same bound) and this in turn implies the condition used in Theorem 9.6. It would be sufficient to show the reverse implication. We develop a fresh approach, however, which crystallizes the ideas underlying the remaining theorems of this section.

Write

$$
\phi(i t)=1-\sum_{n=2}^{\infty} b_{n} n^{-i t}, \quad \phi_{l}(i t)=1-\sum_{n=2}^{l} b_{n} n^{-i t} .
$$

The hypotheses of the theorem imply that $\phi(i t)$ is continuous and

$$
L_{N \rightarrow \infty}\left|\sum_{n=2}^{N} b_{n} n^{-i t}\right| \leqq \theta .
$$

Hence $|\phi(i t)|$ is continuous and bounded from below by a positive constant. It is then easy to see that

$$
M\left\{\phi_{l}(i t) / \phi(i t)\right\} \leqq(1-\theta)^{-2} M\left\{\phi_{l}(i t)\right\}=(1-\theta)^{-2} \sum_{n=2}^{l}\left(b_{n}\right)^{2},
$$

(9.06) $M\left\{1-\phi_{l}(i t) / \phi(i t)\right\}=M\left\{(\phi(i t))^{-1}\left(\phi(i t)-\phi_{l}(i t)\right)\right\} \leqq(1-\theta)^{-2} \sum_{n=l+1}^{\infty}\left(b_{n}\right)^{2}$.

Let

$$
(\phi(z))^{-1}=\sum_{n=1}^{\infty} e_{n} n^{-s}
$$

Then $\left\{e_{n}\right\} \in l_{2}$ since $M\left\{(\phi(i t))^{-1}\right\} \leqq(1-\theta)^{-2}$.

A little reflection shows that the corresponding relations in $x$ are valid when $f_{l}(n x)$ replaces $n^{-z} \phi_{l}(z)$. Thus equation 9.05 implies 


$$
\sum_{n=1}^{\infty} e_{n} f_{l}(n x) \in L_{2}(0 ;-\pi, \pi)
$$

where

$$
f_{l}(x)=\sin x-\sum_{n=2}^{l} b_{n} \sin n x .
$$

Moreover, according to equation 9.06, we have

$$
\left\|\sin x-\sum_{n=1}^{\infty} e_{n} f_{l}(n x)\right\|^{2}=\left\|\sum_{n=1}^{\infty} e_{n}\left(f(n x)-f_{l}(n x)\right)\right\|^{2} \leqq(1-\theta)^{-2} \sum_{n=l+1}^{\infty}\left(b_{n}\right)^{2} .
$$

Obviously this has as a consequence

$$
\left\|\sin x-\sum_{n=1}^{\infty} e_{n} f(n x)\right\|=0 .
$$

This relation remains in force if $x$ is replaced by $k x, k=2,3, \cdots$. Hence $\{\sin n x \mid n=1,2, \cdots\}$ is in the closed linear extension of $\{f(n x)\}$. Accordingly $\{f(n x)\}$ is complete in $L_{2}(0 ;-\pi, \pi)$.

An alternative proof along the same lines depends on the fact that, under the hypothesis $\left\{b_{n}\right\} \in U$, we must have $\left|\sum_{n=2}^{N} b_{n} n^{-i t}\right| \leqq \theta^{\prime}<1$ for all $N>N_{0}$. Hence we can use $M\left\{1-\phi(i t) / \phi_{l}(i t)\right\}$ to give $L_{l+\infty}\left\|\sin x-\sum_{n=1}^{\infty} g_{n}^{l} f(n x)\right\|=0$.

The next two theorems are considerably sharper than Theorem 9.6 and are out of the range of the Paley-Wiener type of result. In a sense these are our strongest completeness results at least when $b_{n} \neq 0$ for incommensurable $n$ values alone. As an incidental, a simple algorithm is developed for determining the $d_{n}$ 's although this is already implicit in the previous theorem. The key to the advance over Theorem 9.6 lies in the following remark. In the latter theorem we required D: the Dirichlet series and the mean $M$ exist for $(\phi(i t))^{-1}$. The proof of Theorems 9.7 and 9.8 pivots on the observation that a formal approximant, $\psi_{l}(i t)$, can be found which, in case $\mathrm{D}$, converges to $(\phi(i t))^{-1}$ or $M(\phi(i t))^{-1}$ when the corresponding limits are taken and such that $L_{l \rightarrow \infty} M \phi(i t) \psi_{l}(i t)$ may exist even when $\mathrm{D}$ is not satisfied. It is somewhat surprising that the coefficient of $\phi(z)$ in equation 9.07 can be taken as $\psi_{l}(z)$.

THEOREM 9.7. If $f(x) \sim \sin x-\sum_{n=2}^{\infty} b_{n} \sin n x, \quad\left\{b_{n}\right\} \in U$, and if (a) $\sum_{(i, j)=1} B_{i, j} \cos (t(\log i-\log j))<1$ a.e., then $\{f(n x)\}$ is complete in $L_{2}(0 ;-\pi, \pi)$.

We shall assume $b_{2} \neq 0$ in the sequel. (The modifications required in the case $b_{2}=0, \cdots, b_{j-1}=0, b_{j} \neq 0$, are obvious.) Write

$$
\phi(z)=1-\sum_{n=2}^{\infty} b_{n} n^{-z}
$$

Then 


$$
(\phi(z))^{-1}=\sum_{n=1}^{\infty} g_{n} n^{-z}=\sum_{r=0}^{\infty}\left(\sum_{n=2}^{\infty} b_{n} n^{-z}\right)^{r}
$$

The expansions converge for sufficiently large $R(z)$ values. It is easy to see that

$$
\phi(z) \sum_{r=0}^{l}\left(\sum_{n=2}^{\infty} b_{n} n^{-z}\right)^{r}=1-\left(\sum_{n=2}^{\infty} b_{n} n^{-z}\right)^{l+1} .
$$

Since $\left\{b_{n}\right\} \in U,|\phi(i t)-1|^{l}$ is a.p. for all positive integers. Hence by the Parseval identity [2, p. 28] the formal expansion as a Dirichlet series of either side of equation 9.07 is valid for $R(z)=0$. The defining condition for a weak $\left\{d_{n}\right\}$ sequence is now turned into a Dirichlet series relation. Thus we have

$$
1-\phi(z) \sum_{n=1}^{M} d_{n} n^{-z}
$$

contains no terms in $m^{-2}$ for $m=1, \cdots, M$. It is easy to see that

$$
1-\phi(z) \sum_{n=1}^{M} g_{n} n^{-z}
$$

has the same property and hence we may take $d_{n}$ as $g_{n}$. Observe now that if the factor multiplying $\phi(z)$ on the left-hand side of equation 9.07 is expanded, we get

$$
\sum_{n=1}^{\infty} g_{n}^{l} n^{-z}
$$

where

$$
L_{l \rightarrow \infty} g_{n}^{l}=d_{n} .
$$

However, we can make the more precise statement

$$
g_{n}^{l}=d_{n}, \quad n=1, \cdots, 2^{l} .
$$

Furthermore

$$
\left\|\sum_{n=1}^{\infty} g_{n}^{l} f(n x)\right\|<\infty
$$

in view of our remarks about the a.p. property of the expressions in equation 9.07.

Manifestly

$$
\left|\sum_{n=2}^{\infty} b_{n} n^{-i \iota}\right|^{2}=\sum_{(i, j)=1} B_{i, j} \cos (t(\log i-\log j)) .
$$

This is almost everywhere less than 1 . Therefore 


$$
M\left\{\left(\sum_{n=2}^{\infty} b_{n} n^{-i t}\right)^{L}\right\} \rightarrow 0
$$

monotonically with $L \rightarrow \infty$. It may be verified at once that

$$
\sin x-\sum_{n=1}^{\infty} g_{n}^{l} f(n x)=\sum_{j=2}^{\infty} h_{j}^{l} \sin j x
$$

where

$$
\sum_{j=2}^{\infty} h_{j}^{l} j^{-2}=\left(\sum_{n=2}^{\infty} b_{n} n^{-8}\right)^{L},
$$

The squared norm of the left-hand side of equation 9.09 is, of course,

$$
\sum_{j=2}^{\infty}\left(h_{j}^{l}\right)^{2}=M\left\{\left(\sum_{n=2}^{\infty} b_{n} n^{-i t}\right)^{L}\right\} .
$$

Hence we have shown that

$$
L_{l \rightarrow \infty}\left\|\sin x-\sum_{n=1}^{\infty} g_{n}^{l} f(n x)\right\|=0 .
$$

We have already seen that such a relation is tantamount to the assertion of completeness of $\{f(n x)\}$.

Remark. We can easily verify that the sequence $\left\{e_{n}\right\}$ in Theorem 9.6 is actually the weak sequence $\left\{d_{n}\right\}$ and belongs to $l_{2}$. However, this latter property is no longer maintained in the theorem above. It seems likely that both of the preceding theorems are valid when the condition $\left\{b_{n}\right\} \in U$ is weakened.

ThEOREM 9.8. If $f(x)=1-\sum_{n=2}^{N} b_{n} \sin n x, b_{j} b_{N} \neq 0, j \neq N$, and if ( $\left.\mathrm{a}^{\prime}\right)$ $\sum_{(i, j)-1} B_{i, j} \cos (t(\log i-\log j)) \leqq 1$, then $\{f(n x)\}$ is complete in $L_{2}(0 ;-\pi, \pi)$.

The case that only a single coefficient in $\left\{b_{n}\right\}$, say $b_{j}$, differs from zero must be excluded. Indeed, in this case one can show immediately that $\left|b_{j}\right|<1$ is a necessary and sufficient condition for completeness.

The demonstration follows the pattern of the previous theorem. The one new element is the proof that for a finite sequence condition $\left(a^{\prime}\right)$ implies condition (a) of Theorem 9.7. This is trivial. Indeed

$$
\sum_{(i, j)=1} B_{i j} \cos (t(\log i-\log j))=1
$$

holds for a denumerable $t$ set, $T$, at best. Suppose, for instance, that a finite $t$-interval contains a nonfinite subset of $T$. Since the left-hand side of equation 9.10 is an entire function, when the restriction to real $t$ values is dropped, it is elementary that then equation 9.10 is an identity. That is to say, $B_{11}=1$ and 
$B_{i j}=0, i j>1$. Then $g(x)=\sum_{n-2}^{N} b_{n} \sin n x /\left(\sum_{2}^{N}\left(b_{n}\right)^{2}\right)^{1 / 2}$ satisfies relation I. However, this is impossible under our hypothesis $b_{j} b_{N} \neq 0$. Indeed, Theorem 2.2 of [1] asserts that no finite collection of $b_{n}$ 's containing at least two nonzero members can satisfy relation $I$.

In the event that there are commensurable values of $n$ for which $b_{n} \neq 0$, the following theorem may be stronger than any of our previous results.

TheOREM 9.9. If $f(x)=\sum_{n=1}^{\infty} b_{n} \sin n x, b_{1} \neq 0,\left\{b_{n}\right\} \in U$, and if $\phi(z)$ $=\sum_{n=1}^{\infty} b_{n} n^{-z}$ has no zeros for $R(z) \geqq 0$, then $\{f(n x)\}$ is complete in $L_{2}(0 ;-\pi, \pi)$.

In view of the a.p. property of $\phi(z)$ it is easy to show that our hypotheses imply that $|\phi(z)|$ has a positive greatest lower bound in $R(z) \geqq 0$. Hence the proof of the theorem can be carried through by making the obvious changes in the demonstration of Theorem 9.6.

In the specialization to systems of the type $\{f(n x)\}$ a result given by Duffin and Eachus [18, Theorem D] amounts to the corollary to Theorem 9.4. The difference between Theorem 9.4 and the corollary may be made apparent even in the case of just three nonvanishing $b_{n}$ 's.

If $\phi(z)=\left(1-b 2^{-z}\right)^{m}, m>1$, Theorem 9.9 applies for all $|b|<1$, but none of the other theorems go so far. The next example in its extreme form falls under Theorems 9.7 and 9.8, but not under any of the other results. It illustrates several matters of interest: for one thing (a) an explicit estimate of the degree of approximation is given. However, the main interest lies in two results which cannot occur in situations covered by the Paley-Wiener theorem cited supra. Thus even though $\{f(n x)\}$ is complete, we show (b) the weak sequence $\left\{d_{n}\right\}$ is not in $l_{2}$ and (c) $\sum_{n=1}^{\infty} d_{n} \sin n x$ is not convergent in the norm topology of $L_{2}(0 ;-\pi, \pi)$.

We take

$$
f(x)=\sin x-b(\sin 2 x+\sin 4 x) .
$$

Then

$$
\phi(z)=1-b\left(2^{-z}+2^{-2 z}\right) .
$$

Manifestly

$$
\left|b\left(2^{-i t}+4^{-i t}\right)\right|^{2 L}=|b|^{2 L}\left(2^{i t / 2}+2^{-i t / 2}\right)^{2 L} .
$$

The problem of computation is turned by the observation that

$$
M\left\{b\left(2^{-i t}+4^{-i t}\right)^{L}\right\}
$$

is simply the constant term in the binomial expansion of the right-hand side of equation 9.12. This is, of course,

$$
|b|^{2 L} \Gamma(2 L+1) /(\Gamma(L+1))^{2}=O\left(|2 b|^{2 L} L^{-1 / 2}\right) .
$$


Hence, with $L=l+1$,

$$
\left\|\sin x-\sum_{n=1}^{N^{l}} g_{n}^{l} f(n x)\right\|=O\left(|2 b|^{2 L} L^{-1 / 2}\right)
$$

and goes to 0 with $l \rightarrow \infty$ if and only if $|b| \leqq 1 / 2$. The case $b=1 / 2$ is comprehended by Theorems 9.7 and 9.8 alone. However, for negative $b$, the values $0>b>-1$ are available by Theorem 9.9 .

With the $f(x)$ of equation 9.10 and $b=1 / 2$ we have

$$
|\phi(i t)|^{-2}=\left(3 / 2-2^{-1} \cos (t \log 2)-\cos (2 t \log 2)\right)^{-1} .
$$

A simple argument depending on Taylor's formula shows immediately that for some positive $\epsilon$ there is a positive constant $h$ independent of $k$ such that the right-hand side of equation 9.14 is larger than $h(t-2 k \pi / \log 2)^{-2}$, $k=0, \pm 1, \cdots$, for $|t-2 k \pi / \log 2| \leqq \epsilon$. It is then easy to see that $M\left\{(\phi(i t))^{-1}\right\}$ $=\infty$. Since $|\phi(s+i t)|$ has a positive lower bound for every $s>0$ it is clear that $M\left\{(\phi(s+i t))^{-1}\right\}$ exists and increases monotonically without limit as $s \rightarrow 0+$. Observe now that

$$
M\left\{(\phi(s+i t))^{-1}\right\}=\sum_{n=1}^{\infty}\left(d_{n}\right)^{2} / n^{2 s} .
$$

Hence $\left\{d_{n}\right\} \notin l_{2}$. This is assertion (b).

The reader will verify that with $d_{n}$ written for the expected $d_{m}, m=2^{n}$,

$$
\sin x-\sum_{n=1}^{N} d_{n} f\left(2^{n} x\right)=d_{N+1} \sin 2^{N+1} x+b d_{N} \sin 2^{N+2} x
$$

where

$$
b^{-1} d_{n+2}=d_{n+1}+d_{n}
$$

Hence we have

$$
d_{n}=\left[\left(b+\left(b^{2}+4 b\right)^{1 / 2}\right)^{n+1}+\left(b-\left(b^{2}+4 b\right)^{1 / 2}\right)^{n+1}\right] /\left(\left(b^{2}+4 b\right)^{1 / 2} 2^{n+1}\right) .
$$

For $b=1 / 2$ we have $d_{n}=(2 / 3)\left(1-(-1 / 2)^{n+1}\right)$. Hence the value of the squared norm of the left-hand side of equation 9.15 approaches $5 / 9$ and, of course, $\sum_{n=2}^{\infty} d_{n} f\left(2^{n} x\right)$ does not converge in the strong $L_{2}(0 ;-\pi, \pi)$ topology. This is assertion (c).

10. A general formulation and a special case. We first give a general setting of considerable interest for the questions occupying us throughout the greater part of this paper. The latter part of the section takes up a particular situation.

We restrict ourselves to $L_{2}(E)$. Thus consider sequences of the type $\left\{f\left(\lambda_{n} x\right)\right\}$. Suppose $\left\{\rho(x) F\left(\lambda_{n} x\right)\right\}, \rho(x) \geqq 0$, is a complete orthonormal set of functions in $L_{2}(E)$. Suppose too that $\lambda_{n} \lambda_{m}=\lambda(m, n)$ where $\lambda(m, n) \in\left\{\lambda_{j}\right\}$. We have then, assuming that all the elements in our analysis are real, 


$$
\begin{aligned}
{\left[F\left(\lambda_{n} x\right), F\left(\lambda_{m} x\right)\right] } & =\int_{E}(\rho(x))^{2} F\left(\lambda_{n} x\right) F\left(\lambda_{m} x\right) d x=\delta_{n, m}, \\
f(x) & \sim \sum_{n=1}^{\infty} \alpha_{n} F\left(\lambda_{n} x\right),
\end{aligned}
$$

and

$$
\begin{aligned}
\left.L f\left(\lambda_{j} x\right), f\left(\lambda_{k} x\right)\right] & =\sum \alpha_{n} \alpha_{m}\left[F\left(\lambda_{i} \lambda_{n} x\right), F\left(\lambda_{k} \lambda_{m} x\right)\right] \\
& =\sum \alpha_{n} \alpha_{m} \delta(\lambda(j, n), \lambda(k, m)) \\
& =\delta_{j, k} .
\end{aligned}
$$

Here $\delta(M, N)=\delta_{M, N}$. (Generalizations to include complex function and parameter values are possible also.) The case treated in this paper and in [1] is that of

$$
\lambda_{n} \lambda_{m}=\lambda_{n m} .
$$

Another "natural" case is that of

$$
\lambda_{n} \lambda_{m}=\lambda_{n+m} .
$$

It is easy to see that this case leads to

V

$$
\sum_{n=0}^{\infty} \alpha_{n} \alpha_{n+k}=\delta_{0, k}
$$

We show presently that this is essentially a special case of the theory already developed. No other cases will be treated in this paper.

THEOREM 10.1. There is an isomorphism between the set of solutions of $\mathrm{V}$ and those of II with a one-element base.

We understand by this isomorphism that there is a 1-1 correspondence between the solutions referred to and also topological equivalence in $l_{2}$. This will be clear from the simple proof. For convenience denote solutions of $\mathrm{V}$ in $l_{2}$ by $\left\{\alpha_{N}\right\} \in J$. Let $p$ be a prime. Let $n=p^{N}, N=0,1,2, \cdots$. Associate the sequences $\left\{a_{n}\right\}$ and $\left\{\alpha_{N}\right\}$ according to the relations

$$
a_{n}=\alpha_{N} \text {. }
$$

It is easy to see that if $\left\{a_{n}\right\} \in K$ then $\left\{\alpha_{N}\right\} \in J$. Conversely, $\left\{\alpha_{N}\right\} \in J$ implies $\left\{a_{n}\right\} \in K$. Moreover the map defined by equation 10.01 is order-preserving on the ordinals used as subscripts. Hence, plainly, the map is a topological isomorphism.

Accordingly every result on sequences $\left\{a_{n}\right\} \in K$ with a one-element base may be paraphrased to yield a result concerned $\left\{\alpha_{N}\right\} \in J$ (and conversely). From the viewpoint of the associated functions, the relation is that between $\phi(z)$ $=\psi\left(p^{-z}\right)$ and $\psi(w)$ where $w=p^{-z}$. Thus, for instance, relation III becomes 


$$
\psi(w) \psi\left(w^{-1}\right)=1
$$

and $M\{\phi(z)\}$ is now

$$
\frac{1}{2 \pi} \int_{-\pi}^{\pi}\left|\psi\left(r e^{i \theta}\right)\right|^{2} d \theta
$$

ThEOREM 10.2. Let $B$ be the class of all functions regular in $|w|<1$ and of modulus not greater than 1 there. Let $S_{n}(w)$ be the sum of the first $n$ terms of the Maclaurin series. Then $S_{n}(w)$ takes on its maximum value for a quasi-elementary solution of $J$, namely

$$
w^{n} K_{n}\left(w^{-1}\right) / K_{n}(w), \quad K_{n}(w)=\sum_{j=1}^{n}\left(\Gamma(2 j) / 2^{i} \Gamma(j+1)\right) w^{j} .
$$

This result is a well known theorem of Landau's [19, p. 26]. (Actually the Landau theorem states the numerical value of the bound for $\left|S_{n}(w)\right|$ and does not mention $K_{n}(w)$, but this function comes into Landau's proof.)

Remark. This theorem suggests the possibility of characterizing solutions in $K$ by extremal properties for more general bases.

Theorem 10.3. (A) If $\phi(z)=K$ has a one-element base, then $L_{s \rightarrow 0+} \phi(s+i t)$ $=\phi(i t)$ exists and the limit function has the modulus 1 almost everywhere.

Let

$$
\phi(z)=\sum_{N=0}^{\infty} \alpha_{N} p^{-n z}=\psi\left(p^{-s}\right)
$$

Then

$$
w=p^{-s}
$$

maps the strip $0 \leqq I_{m}(z)<2 \pi / \log p, R(z) \geqq 0$, conformally onto the circle $|w| \leqq 1$.

Since $\phi(z)$ is periodic with period $2 \pi / \log p$ it is clear that $\psi(w)=\sum_{N-\infty}^{\infty} \alpha_{N} w^{N}$ is analytic with $|\psi(w)|<1$ in $|w|<1$. The Fatou theorem type of argument leads, just as in the case of Theorem 4.6, to

$$
\begin{aligned}
L_{r \rightarrow 1} \psi\left(r e^{i 0}\right) & =\psi\left(e^{i t}\right) \quad \text { a.e., } \\
\left|\psi\left(e^{i v}\right)\right| & \leqq 1, \\
L_{s \rightarrow 0+} \phi(s+i t) & =\phi(i t) \quad \text { a.e. }
\end{aligned}
$$

Now, however, we can make an advance by using the fact that

$$
\frac{1}{2 \pi} \int_{-\pi}^{\pi}\left|\psi\left(e^{i \theta}\right)\right|^{2} d \theta=\sum_{N=0}^{\infty}\left(\alpha_{N}\right)^{2}=\sum_{n=1}^{\infty}\left(a_{n}\right)^{2}=1 .
$$

Evidently equations 10.04 and 10.05 imply 
(10.06)

$$
\left|\psi\left(e^{i \theta}\right)\right|=1 \quad \text { a.e. }
$$

and therefore

$$
|\phi(i t)|=1 \quad \text { a.e. }
$$

We have incidentally established that if $\psi(w) \in J$ then $\psi(w)$ takes on boundary values of modulus 1 a.e. as $r \rightarrow 1$.

THEOREM 10.4. If $\phi(z)$ has a one-element base, then a necessary and suffcient condition that $\phi(z) \in K$ is that $H(\lambda)$ satisfy $\mathrm{W}$.

The necessity part is merely Theorem 7.3. To prove sufficiency we remark first that the relation $W$ implies that $\phi(i t)$ exists with $|\phi(i t)|=1$ a.e. Moreover, in view of Theorem $7.4(\mathrm{C})$, we easily infer that $L_{s \rightarrow 0+} \phi(s+i t) \mid=\phi(i t)$ in the norm topology over any finite $t$ interval. However, since there is a one-element base, $\phi(s+i t)$ and $\phi(i t)$ must be periodic. Thus write $\phi(z)=\sum_{N=0}^{\infty} \alpha_{N} p^{-n z}$. Hence

$$
M\{\phi(s+i t)\}=(2 \log p)^{-1} \int_{-\log p}^{\log p}|\phi(s+i t)|^{2} d t
$$

and the limit as $s \rightarrow 0+$ on the left-hand side yields

$$
\frac{1}{2 \log p} \int_{-\log p}^{\log p}|\phi(i t)|^{2} d t=1 .
$$

The conditions of Theorem 6.1 are satisfied. Hence $\phi(z) \in K$.

If $\psi(w)$ is regular and bounded in $|w|<1$ and satisfies equation 10.06 then it is a simple matter to show that $\psi(w) \in J$. This observation allows us to exhibit the most general such function. Indeed we need merely slightly modify a result of Seidel's [20, p. 204] to get

$$
\begin{gathered}
\psi(w)=B(w) \exp \frac{1}{\pi} \int_{0}^{\pi}\left(1-|w|^{2}\right) /(1-2|w| \cos (\arg w-\theta) \\
\left.+|w|^{2}\right) d \sigma(\theta) .
\end{gathered}
$$

Here $B(w)$ is the Blaschke product $\Pi\left(A_{i}-w\right) /\left(1-\bar{A}_{i} w\right)$ with either real or conjugate complex pairs of zeros within the unit circle subject to $\prod\left|A_{i}\right|>0$ and $\sigma(\theta)$ is monotone non-increasing with vanishing derivative a.e. Hence setting $w=m^{-z}$ yields the most general solution on a one-element base.

Added in proof. The writer is indebted to Dr. R. P. Boas for a reference to a significant paper by A. Wintner, Diophantine approximations and Hilbert's space, Amer. J. Math. vol. 66 (1944) pp. 564-578. The reader will find this work of interest in connection with the ideas of $\$ 9$.

\section{BIBLIOGRAPHY}

1. D. G. Bourgin and C. W. Mendel, Orthonormal sets of periodic functions of the type $\{f(n x)\}$, Trans. Amer. Math. Soc. vol. 57 (1945) pp. 332-363. 
2. A. S. Besicovitch, Almost periodic functions, Cambridge University Press, 1932.

3. H. Bohr, Über die Bedeutung der Potenzreihen unendlich vieler Variabeln in der Theorie der Dirichletschen Reihen, Nachr. Ges. Wiss. Göttingen (1913) pp. 441-488.

4. O. Toeplitz, $Z u$ Theorie der Dirichlet Reihen, Amer. J. Math. vol. 60 (1938) pp. 880-888.

5. A. Wintner, Spektratheorie der unendlichen Matrizen, Leipzig, 1929.

6. L. Bieberbach, Moderne Funktionentheorie, 2d ed., Teubner, 1931.

7. E. C. Titchmarsh, The theory of functions, 2 d ed., Oxford University Press, 1939.

8. S. Banach, Théorie des opérations linéaries, Warsaw, 1932.

9. P. Fatou, Serie trigonometrique et serie de Taylor, Acta Math. vol. 30 (1906) pp. 335-400.

10. F. Riesz and M. Riesz, Über die Randwerte eines analytischen Funktion. Compte rendu au quatrième Congrès des mathematiciens Scandinaves, Stockholm, pp. 27-47.

11. W. F. Osgood, Lehrbuch der Funktionentheorie, vol. 2, Teubner, 1924. 199.

12. G. N. Watson, General transforms, Proc. London Math. Soc. vol. 35 (1933) pp. 156-

13. D. V. Widder, The Laplace transform, Princeton University Press, 1941.

14. O. Szász, Über die Approximation stetiger Funktionen durch gegebene Funktionenfolgen, Math. Ann. vol. 104 (1931) pp. 155-160.

15. R. E. A. C. Paley and N. Wiener, Fourier transforms in the complex plane, Amer. Math. Soc. Colloquium Publications, vol. 19, 1934.

16. D. G. Bourgin, Closure of products of functions, Bull. Amer. Math. Soc. vol. 46 (1940) pp. 807-815. 100.

17. O. Frink, Series expansions in linear vector spaces, Amer. J. Math. vol. 63 (1941) pp. 87-

18. R. J. Duffin and J. J. Eachus, Some notes on an expansion theorem of Paley and Wiener, Bull. Amer. Math. Soc. vol. 48 (1942) pp. 850-855.

19. E. Landau, Ergebnisse der Funktionentheorie, 2d ed., Springer, 1929.

20. W. Seidel, On the distribution of values of bounded analytic functions, Trans. Amer. Math. Soc. vol. 36 (1934) pp. 201-226.

UNIVERSITY OF ILLINOIS, URBANA, ILL. 